\title{
Frequency-shift vs phase-shift characterization of in-liquid quartz crystal microbalance applications
}

\author{
Y. J. Montagut, ${ }^{1}$ J. V. García, ${ }^{1}$ Y. Jiménez, ${ }^{1}$ C. March, ${ }^{2}$ A. Montoya,${ }^{2}$ and A. Arnau ${ }^{1, a)}$ \\ ${ }^{1}$ Grupo de Fenómenos Ondulatorios, Departamento de Ingeniería Electrónica, Universitat Politècnica \\ de València, Spain \\ ${ }^{2}$ Instituto Interuniversitario de Investigación en Bioingeniería y Tecnología Orientada al Ser Humano, \\ Universitat Politècnica de València, Spain
}

(Received 16 March 2011; accepted 16 May 2011; published online 10 June 2011)

\begin{abstract}
The improvement of sensitivity in quartz crystal microbalance (QCM) applications has been addressed in the last decades by increasing the sensor fundamental frequency, following the increment of the frequency/mass sensitivity with the square of frequency predicted by Sauerbrey. However, this sensitivity improvement has not been completely transferred in terms of resolution. The decrease of frequency stability due to the increase of the phase noise, particularly in oscillators, made impossible to reach the expected resolution. A new concept of sensor characterization at constant frequency has been recently proposed. The validation of the new concept is presented in this work. An immunosensor application for the detection of a low molecular weight contaminant, the insecticide carbaryl, has been chosen for the validation. An, in principle, improved version of a balanced-bridge oscillator is validated for its use in liquids, and applied for the frequency shift characterization of the QCM immunosensor application. The classical frequency shift characterization is compared with the new phase-shift characterization concept and system proposed. (C) 2011 American Institute of Physics. [doi:10.1063/1.3598340]
\end{abstract}

\section{INTRODUCTION}

Acoustic sensing has taken advantage of the progress made in the last decades in piezoelectric resonators for radio-frequency (RF) telecommunication technologies. The so-called gravimetric technique is based on the change in the resonance frequency experimented by the resonator due to a mass attached on the sensor surface; ${ }^{1}$ it has opened a great deal of applications in bio-chemical sensing in both gaseous and liquid media. ${ }^{2-10}$ This characteristic allows using the gravimetric techniques based on acoustic sensors for a label-free and a quantitative time-dependent detection.

The classical quartz crystal microbalance (QCM) has been the most used acoustic device for sensor applications. However, other acoustic devices such as surface generated acoustic wave (SGAW) (Ref. 4) and film bulk acoustic resonators (FBAR) (Refs. 8 and 11-14) have been, and are being used, for the implementation of nano-gravimetric techniques due to the feasibility of obtaining much higher resonant frequency in these devices than in classical QCM resonators. The absolute frequency/mass sensitivity, given by the ratio between the resonant frequency-shift $\Delta f$ and the surface mass density shift $\Delta \mathrm{m}: S_{a}=\Delta f / \Delta m$, theoretically increases with the square of the fundamental frequency; ${ }^{1}$ absolute sensitivities of a $30 \mathrm{MHz}$ QCM reach $2 \mathrm{~Hz} \mathrm{~cm}^{2} \mathrm{ng}^{-1}$, with typical mass resolutions around $10 \mathrm{ng} \mathrm{cm}{ }^{-2} \cdot{ }^{15} \mathrm{~A}$ resolution improvement down to $1 \mathrm{ng} \mathrm{cm}{ }^{-2}$ seems to be feasible by optimizing the characterization electronic interface as well as the fluidic system. Consequently, much higher sensitivity

\footnotetext{
a) Author to whom correspondence should be addressed. Electronic mail: aarnau@eln.upv.es.
}

is expected at higher resonant frequencies. However, the increase in frequency-shift/mass sensitivity has not been paired with the expected improvement in terms of limit of detection (LOD). Effectively, thin film electroacoustic technology has made possible to fabricate quasi-shear mode thin FBAR, operating with a sufficient electromechanical coupling for being used in liquid media at $1-2 \mathrm{GHz} ;{ }^{12,16}$ however, the higher frequency and the smaller size of the resonator result in that the boundary conditions have a much stronger effect on the FBAR performance than on the QCM response. A higher mass sensitivity is attained, but with an increased noise level as well, thus moderating the gain in resolution. ${ }^{13,17}$ So far only publications of network analyzer based FBAR sensor measurements have been published in the literature, which show that the FBAR mass resolution is very similar if not better than for oscillator based QCM sensors. ${ }^{14,18}$ On the other hand, the mass sensitivity of Love mode SGAW sensors has been evaluated. ${ }^{19-21}$ Kalantar and coworkers reported a sensibility of $95 \mathrm{~Hz} \mathrm{~cm} \mathrm{ng}^{-1}$ for a $100 \mathrm{MHz}$ Love mode sensor, which is much better than the typical values reported for low frequency QCM technology. ${ }^{22}$ However, Moll and coworkers reported a LOD for a Love sensor of $400 \mathrm{ng} \mathrm{cm}^{-2}$, this reveals once again that an increase in the sensitivity does not mean, necessarily, an increase in the LOD. ${ }^{23}$ Moreover, these results have been compared with typical $10 \mathrm{MHz}$ QCM sensors; recently, an electrodeless QCM biosensor for $170 \mathrm{MHz}$ fundamental frequency, with a sensitivity of $67 \mathrm{~Hz} \mathrm{~cm}{ }^{-2} \mathrm{ng}^{-1}$, has been reported; ${ }^{24}$ this shows that the classical QCM technique still remains as a promising technique. The main challenges remain on the improvement of the sensitivity, but with the aim of getting a higher mass resolution, multi-analysis, and integration capabilities and reliability. 
This analysis makes clear that increasing the resonant frequency of the sensor is not the only aspect to keep in mind for resolution improvement; the configuration setup has an important role, including the fluidic system and electronic characterization interface. Once all the care has been taken in optimizing the fluidic aspects, the role of the electronic interface is of maximum relevance.

In practice, focusing on in-liquid QCM applications, the sensor characterization techniques provide, among other relevant parameters, the resonance frequency shift of the sensor: ${ }^{25,26}$ network or impedance analysis is used to sweep the resonance frequency range of the resonator and determine the maximum conductance frequency, ${ }^{27,28}$ which is almost equivalent to the motional series resonance frequency of the resonator-sensor; impulse excitation and decay method techniques are used to determine the series-resonance or the parallel-resonance frequency depending on the measuring setup; ${ }^{29,30}$ oscillator techniques are used for a continuous monitoring of a frequency which corresponds to a specific phase shift of the sensor in the resonance bandwidth, ${ }^{31-35}$ this frequency can be used, in many applications, as reference of the resonance frequency of the sensor; and the lock-in techniques, which can be considered as sophisticated oscillators, are designed for a continuous monitoring of the motional series resonance frequency or the maximum conductance frequency of the resonator-sensor. ${ }^{36-42}$ In order to assure that the frequency shift is the only parameter of interest, a second parameter providing information of the constancy of the properties of liquid medium is important, for instance, in piezoelectric biosensors; this parameter depends on the characterization system being: the maximum conductance or the conductance bandwidth in impedance analysis, the dissipation factor in decay methods, and a voltage associated with the sensor damping in oscillator techniques.

For high frequency resonators only impedance analysis provides accurate results, but its high cost and large dimensions prevent its use for sensor applications. Consequently, oscillators are taken as alternative for sensor resonance frequency monitoring; the low cost of their circuitry as well as the integration capability and continuous monitoring are some features which make the oscillators to be the most common alternative for high resonance frequency QCM sensors. However, in spite of the efforts carried out to design oscillator configurations suitable for in-liquid applications ${ }^{43-51}$ the poor stability of high frequency QCM systems based on oscillators has prevented increasing the limit of detection despite the higher sensitivity reported. ${ }^{52-56}$

A higher sensitivity is necessary when a higher resolution is required. This happens in those applications where very tiny changes in the sensor resonant frequency, due to the perturbation process to be monitored, are expected. These very small frequency shifts, in the order of tens of hertz, and mainly due to a mass transfer effect over different kind of coatings with different contacting media, must be monitored under very different damping conditions which depend on the physical and geometrical properties of the coating and contacting media. For instance, in piezoelectric biosensors the coating can be considered, in general, like an acoustically thin layer and the contacting media is a water-like solution which provides a relatively small damping on the sensor. On the contrary, in other cases like, for example, in some electrochemical applications where polymer coatings are involved, these tiny frequency shifts must be monitored under much higher damping conditions. Continuous monitoring of very small frequency shifts at very high resonance frequencies can be easily performed with well-designed oscillators; however, when the quality factor of the resonator-sensor is relatively low, circuits able to oscillate under these special conditions have to be performed.

A phase-shift monitoring at a constant frequency in the sensor resonance bandwidth has been recently proposed as an alternative characterization method for high resolution QCM applications. ${ }^{57}$ In the present article this alternative method is validated in a real application, and compared with an, in principle, improved version of a balanced-bridge oscillator. ${ }^{25,51}$ The comparison is made with relatively low frequency sensors $(10 \mathrm{MHz})$, where the performance of the oscillator circuit can be considered nearly ideal.

\section{THEORETICAL ASPECTS}

For a great deal of in-liquid QCM applications, as it is the case of QCM biosensors, Martin's equation (Eq. (1)) is generally applied, ${ }^{58}$ which combines the additive contribution of the mass effect (Sauerbrey ${ }^{1}$ ) and the liquid effect (Kanazawa): ${ }^{59}$

$$
\Delta f=-\frac{2 f_{o}^{2}}{Z_{c q}}\left(m_{c}+m_{L}\right) .
$$

In the former equation, $f_{o}$ is the fundamental resonant frequency, $Z_{c q}$ is the characteristic acoustic impedance of the quartz, $m_{c}$ is the surface mass density of the coating and $m_{L}$ $=\rho_{L} \delta_{L} / 2$ where $\rho_{L}$ and $\delta_{L}=\left(\eta_{L} / \pi f_{o} \rho_{L}\right)^{1 / 2}, \eta_{L}$ being the liquid viscosity, are respectively, the liquid density and the wave penetration depth of the acoustic wave in the liquid: $m_{L}$ is, in fact, the equivalent surface mass density of the liquid, which moves in an exponentially damped sinusoidal profile, due to the oscillatory movement of the surface of the sensor.

According to Eq. (1), the frequency shift, associated with a certain mass change, increases directly proportional to the square of the fundamental resonance frequency. Consequently, the most relevant parameter used up to date for the characterization of microbalance sensors has been the sensor resonance frequency-shift. However, the great efforts performed to improve the sensitivity of the sensor are useless if they are not accompanied with an increase in the limit of detection. As mentioned, the increase of the sensor frequency has not carried a parallel improvement in the mass resolution. Effectively, the sensitivity will not be improved if the frequency stability is not improved as well. In oscillators, the origin of the frequency instability is the phase instability, ${ }^{25,57}$ and a direct relationship can be obtained between a phase shift and the corresponding frequency shift, through the definition of the stability factor $S_{F}$ of a crystal resonator operating at its series resonance frequency $f_{o}$ :

$$
S_{F}=\frac{\Delta \varphi}{\Delta f} f_{o}=2 Q
$$


where $\Delta f$ is the frequency shift necessary to provide a phase shift $\Delta \varphi$ in the phase-frequency response of the resonator, around $f_{o}$, and $Q$ is the series quality factor of the resonator.

According to Eq. (2) the frequency noise $\Delta f_{n}$ associated with a phase noise in the circuitry $\Delta \varphi_{n}$ is

$$
\Delta f_{n}=\frac{f_{o}}{2 Q} \Delta \varphi_{n} .
$$

Consequently, because the quality factor of the unperturbed resonator is normally reduced proportionally to $1 / f_{o}$, the frequency instability is increased in relation to the square of frequency. Moreover, the phase response of the electronic components of an oscillator gets worse with increasing the frequency, which increases, even more, the noise. Furthermore, if the limit of the detection is assumed to be three times the level of noise $\left(\Delta \varphi_{\min }=3 \Delta \varphi_{n}\right)$, the minimum detectable surface mass density change of a QCM, according to the definition of the absolute frequency/mass sensitivity $S_{a}$ and Eq. (3), will be

$$
\Delta m_{\min }=\frac{f_{o}}{2 Q S_{a}} \Delta \varphi_{\min }
$$

The former equation seems to indicate that for a given minimum detectable phase of the measuring system, the surface mass limit of detection does not depend on the frequency. Fortunately this is not completely true; the liquid medium has not been taken into account in the obtaining of the previous equation.

\section{FREQUENCY-SHIFT VS PHASE-SHIFT}

Following a similar mathematical development described elsewhere ${ }^{57}$ the next generalized approximated equation for the phase-shift of a signal, of constant frequency very close to the motional series resonant frequency of the resonatorsensor, to small changes both in the coating mass and liquid properties, is found:

$$
\Delta \varphi(\mathrm{rad})=-\frac{\Delta m_{c}+\Delta m_{L}}{m_{q}+m_{L}} .
$$

In the former equation $m_{q}=Z_{c q} / 4 f_{o} Q_{o}$ and $m_{L}$, previously defined in Eq. (1), can be written as follows $m_{L}$ $=Z_{c q} / 4 f_{o} Q_{L}$, where $Q_{o}=\mathrm{c}_{66} / \omega_{0} \eta_{q}$ is the series quality factor of the unperturbed resonator, $\mathrm{c}_{66}$ and $\eta_{\mathrm{q}}$ being, respectively, the shear modulus and the effective viscosity of the quartz crystal, and $\omega_{\mathrm{o}}$ the angular resonant frequency; and $Q_{L}$ is the series quality factor of the resonator under liquid loading conditions, which is given by the following equation:

$$
Q_{L}=\frac{Z_{c q} \sqrt{\pi}}{2} \frac{1}{\sqrt{f_{o}}} \frac{1}{\sqrt{\rho_{L} \eta_{L}}} .
$$

In many in-liquid QCM applications $m_{L}$ can be assumed to be constant and in most of them $m_{q} \ll m_{L}$; thus Eq. (5) reduces to

$$
\Delta \varphi \approx-\frac{\Delta m_{c}}{m_{L}},
$$

which was previously obtained by the authors elsewhere. ${ }^{57}$
According to Eq. (6) the limit of mass-change detection $\Delta m_{\min }$ corresponding to the phase-shift detection limit of the system $\Delta \varphi_{\min }$ will be given by: $\Delta m_{\min } \approx-m_{L} \Delta \varphi_{\min }$. Consequently, the mass resolution increases $\left(\Delta m_{\min }\right.$ decreases) with the decrease of $m_{L}$; therefore, because $\mathrm{m}_{L}$ decreases proportionally to $1 / f^{1 / 2}$, the resolution in the detection of surface mass density changes increases with $f^{1 / 2}$ for a given $\Delta \varphi_{\min }$.

This is not in contradiction with Eq. (4); simply the effective reduction of the quality factor of the sensor is proportional to $1 / f^{1 / 2}$ instead of to $1 / f$ when the contacting liquid is considered. This is not true in air because the approximation $m_{q} \ll m_{L}$ made in Eq. (5) to obtain Eq. (6) is not acceptable. In air, an increase in frequency does not improve the limit of detection unless the stability and the phase detection limit of the measuring system are improved. Curiously, this also happens when only changes in the liquid properties occur. Effectively, when the aim is to monitor changes in the properties of the liquid in contact with the sensor: $\Delta\left(\eta_{L} \rho_{L}\right)^{1 / 2}$, the phase shift related to these changes is, according to Eq. (5), given by

$$
\Delta \varphi \approx-\frac{\Delta m_{L}}{m_{L}}=-\frac{\Delta \sqrt{\rho_{L} \eta_{L}}}{\sqrt{\rho_{L} \eta_{L}}} .
$$

Therefore, it is not possible to increase the resolution in the detection of changes in the liquid properties by increasing the frequency. According to the previous considerations, it is important to check the limits for which the approximation $m_{q}$ $\ll m_{L}$ is acceptable:

The parameter $m_{q}$ is independent of the frequency, and a reference value of $m_{q}=2.2 \cdot 10^{-6} \mathrm{~kg} \mathrm{~m}^{-2}$ is obtained for a real $10 \mathrm{MHz}$ AT-cut quartz crystal sensor with a typical $Q_{o}$ around $10^{5}$ (see definition below Eq. (5) with $Z_{c q}=8.838833$ $10^{6} \mathrm{~N} \mathrm{~s} \mathrm{~m}^{-3}$ ). The approximation can be considered acceptable for ratios of $m_{q} / m_{L} \leq 0.1$. This ratio is given by

$$
\frac{m_{q}}{m_{L}}=2.2 \cdot 10^{-6} \sqrt{4 \pi} \frac{\sqrt{f}}{\sqrt{\rho_{L} \eta_{L}}} \leq 0.1 .
$$

The former equation indicates that for a given liquid the ratio $m_{q} / m_{L}$ only depends on the frequency; the worst case occurs for low density-viscosity liquids like, for instance, water where $\eta_{L} \rho_{L}=1$. In this case the maximum frequency for which the approximation $m_{q} \ll m_{L}$ is acceptable is around $165 \mathrm{MHz}$. Moreover, Eq. (5) also indicates that when $m_{L}$ decreases to a value much smaller than $m_{q}$, no further improvement in the resolution can be obtained by increasing the frequency; this happens for ratios $m_{q} / m_{L} \geq 0.9$ which are obtained, under the previous conditions, for frequencies higher than $10 \mathrm{GHz}$.

The previous analysis allows concluding the following important remarks: (1) the sensitivity of a QCM always increases with increasing the frequency; however, the mass resolution, which is the parameter of interest, only increases with the frequency if the noise is, at least, maintained constant or reduced. Moreover, this increase in the mass resolution is only valid for in-liquid QCM and not for in-gas QCM; and (2) once all the cares have been taken into account to reduce the perturbations on the resonator-sensor such as temperature and pressure fluctuations, etc., the mass resolution is only depending 


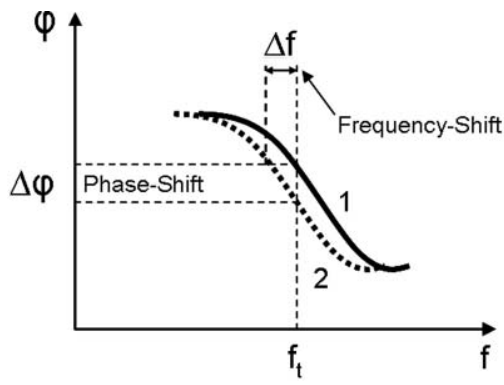

(a)

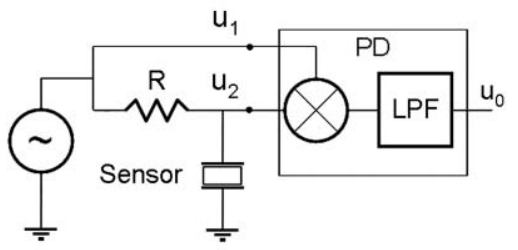

(b)

FIG. 1. (a) Description of the phase-shift characterization versus the frequency-shift method, (b) implementation block diagram.

on the interface system, its stability, and its phase detection limit.

Consequently, unlike in oscillators for RF applications, in oscillators based on QCM sensors, the resonator is not included in the circuitry with the aim of stabilizing the oscillator system, although evidently it does it; just on the contrary, the oscillator circuitry should be as ideal as possible for not influencing the shifts in the sensor phase due to the monitoring processes. Unfortunately, the implementation of an ideal oscillator for high frequency QCM sensors, keeping in mind the low quality factors reached by these sensors under liquid conditions, and the very low phase noise that it is necessary, is not an easy task.

By keeping in mind the previous considerations, a different approach was recently proposed: ${ }^{57}$ taking into account that the expected frequency shifts in those QCM applications where a high resolution is necessary, for example, in biosensors, are very small, it could be possible to interrogate the sensor with an appropriate constant frequency signal, in the sensor resonance bandwidth, and then measure the change in the phase response of the sensor, while maintaining the frequency of the testing signal in the resonance bandwidth; Fig. 1(a) depicts the frequency-shift versus the phase-shift characterization methods.

A similar approach has been already applied under different conditions by some authors. ${ }^{60,61}$ The advantage of this approach is that the sensor is interrogated with an external source which can be designed to be very stable and with extremely low phase and frequency noises, even at very high frequencies. Moreover, a very simple circuit can be used for the phase-mass characterization approach as depicted in Fig. 1(b), where a mixer based phase detector is used. In the next paragraph two practical systems are described which will be useful for validating the phase-shift against the frequencyshift characterization. An improved version of the balancedbridge oscillator proposed elsewhere ${ }^{25}$ is extensively tested under different damping conditions showing the effects of the

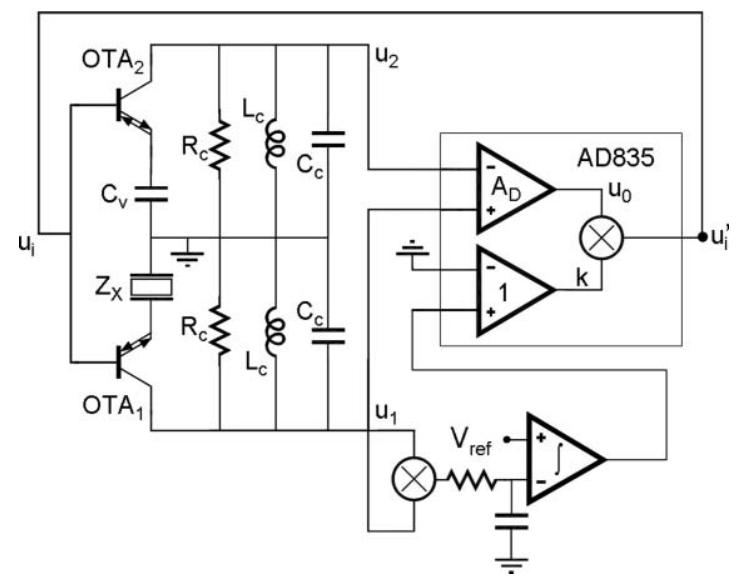

FIG. 2. Schematics of the balanced-bridge oscillator proposed.

non-ideal behaviour of oscillators to monitor the frequencyshifts under different damping conditions. Nonetheless, for constant damping conditions and low frequency sensors (10 MHz AT-cut quartz resonators), the oscillator can be used for resonance frequency shift monitoring and therefore be used to validate the phase-shift method by comparison of the results.

\section{DESCRIPTION OF THE SYSTEMS}

\section{A. Improved balanced-bridge oscillator}

The proposed interface is shown in Fig. 2, and is based on the balanced-bridge oscillator presented elsewhere, ${ }^{51}$ where the transistors have been replaced by operational transconductance amplifiers or diamond transistors (OTA 1-2). The mixer circuit based on the integrated circuit (IC) AD835, with two differential inputs, allows the implementation of a differential amplification with automatic gain control (AGC), which minimizes the nonlinearities of the active devices and provides information about the sensor damping. This configuration allows, in principle, a parallel capacitance compensation which ideally provides oscillation at zero-phase loop condition; the parallel circuit $L_{C}-C_{C}$ is included to drastically reduce the loop-gain for undesired frequencies. For ideal components and parallel capacitance compensation the oscillation frequency should be the motional series resonant frequency of the sensor under different damping conditions, and the AGC voltage would provide information about the resonator motional resistance.

Effectively, the input voltage $u_{i}$ is transferred to the emitters and the emitter currents are non-inverted voltageconverted to the collectors into $u_{1}$ and $u_{2}$ as follows:

$$
\begin{gathered}
u_{1}=u_{i} Y_{X} Z_{C}, \\
u_{2}=u_{i} Y_{C v} Z_{C},
\end{gathered}
$$

where $Y_{X}=j \omega C_{o}+1 / Z_{m}$ is the admittance of the QCM sensor formed by the so-called static capacitance $C_{o}$ in parallel with the so-called motional branch whose impedance $Z_{m}$ is formed by a $R_{m}, L_{m}, C_{m}$ series equivalent circuit, being $Z_{m}=R_{m}$ $+j\left(L_{m} \omega-1 / \omega C_{m}\right) ; Y_{C v}=j \omega C_{v}, Z_{C}=R_{C}+j\left(L_{C} \omega-1 / \omega C_{C}\right)$ and $\omega$ is the angular frequency. 
The voltages at the collectors are differentially amplified with one of the high input impedance differential amplifiers of the AD835, and the output signal is level controlled with a multiplier giving the following output signal $u_{i}^{\prime}$ which is fed-back to the input:

$$
u_{i}^{\prime}=A_{D} k\left(\frac{1}{Z_{m}}+j \omega\left(C_{o}-C_{v}\right)\right) Z_{C} u_{i}
$$

Because $u_{i}^{\prime}=\mathrm{u}_{\mathrm{i}}$ the final loop condition results:

$$
A_{D} k\left(\frac{1}{Z_{m}}+j \omega\left(C_{o}-C_{v}\right)\right) Z_{C}=1 .
$$

Under ideal conditions, it is assumed that the parallel circuit $Z_{C}$ has been designed to resonate at the oscillation frequency, therefore $Z_{C} \approx R_{C}$; the parallel capacitance has been compensated, $C_{o}=C_{V}$; and the OTAs and the multiplier do not produce phase-shifts, it is to say $A_{D}$ and $k$ are real numbers. Consequently, the loop-phase condition given by Eq. (11) indicates that, under the previous conditions, the oscillation frequency corresponds to the motional series resonant frequency at which $Z_{m}=R_{m}$, and the loop-gain at the oscillation frequency reduces to

$$
A_{D} k \frac{R_{C}}{R_{m}}=1 .
$$

Therefore the automatic gain control voltage, $k$ $=R_{m} / A_{D} R_{C}$, is proportional to the value of the motional resistance $R_{m}$ for given values of the differential gain $A_{D}$ and the resistance $R_{C}$. The objective of the AGC is to maintain constant the amplitude of the signal $u_{1}$; with this purpose a dc signal, associated with the amplitude of the sinusoidal signal $u_{1}$, is obtained by low pass filtering the output of a multiplier whose inputs are connected to $u_{1}$.

Experimental results will provide the level of fulfilment of the previous equations regarding the degree of ideal performance of the components of the oscillator.

\section{B. Phase-shift characterization interface}

A schematic interface for the phase-shift characterization method was proposed elsewhere ${ }^{57}$ and it has been implemented now for its validation. The core of the interface is the sensor circuit which is depicted in Fig. 3. Two parallel branches form a differential circuit. Because the testing signal $u_{t}$ has constant frequency $f_{t}$, the only element in the circuit which contributes to a change in the phase shift between the reference signal $u_{1}$ and the signal $u_{2}$ is the change in the phase-frequency response due to the sensor perturbation. Therefore, this phase-shift can be continuously monitored by a phase-detector. The IC AD8302 from Analog Devices has been used for this purpose; it includes a mixer and the lowpass filter (LPF), connected in series behind the signals $u_{1}$ and $u_{2}$, which act as a phase detector for small phase-shifts around $90^{\circ}$ between the input signals. ${ }^{62}$ Thus, for a proper operation it is convenient to phase-shift $90^{\circ}$ the testing signals in each branch of the sensor circuit; for this purpose the networks formed by $R_{i}$ and $C_{i}$ at the inputs of the sensor circuit have been included. The phase-shifting networks formed by $R_{i}$ and $C_{i}$ must be designed coherently with the resonant frequency of

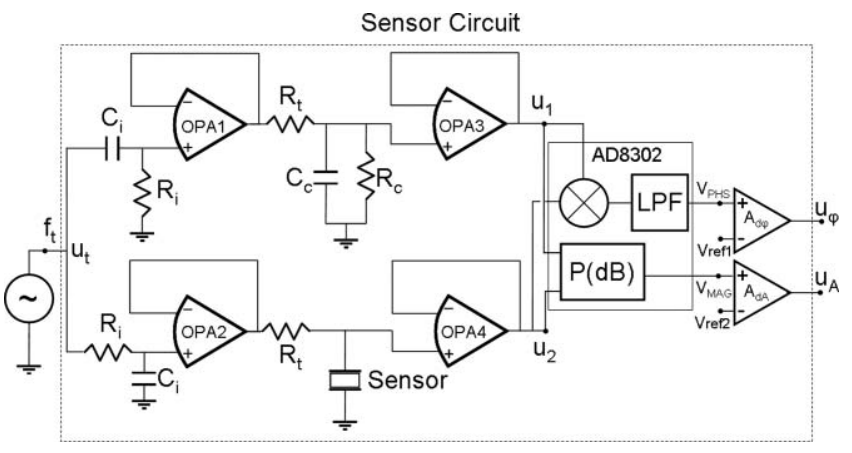

FIG. 3. Schematics of the phase-shift characterization system.

the sensor in order to obtain two signals $90^{\circ}$ phase-shifted and of similar amplitude. The IC-AD8302 additionally includes a block, formed by logarithmic amplifiers, which provides a voltage proportional to the decibel ratio of the input signals $u_{1}$ and $u_{2}$. Both the phase detector and the logarithmic amplifier block have responses $V_{\mathrm{PHS}}$ and $V_{\mathrm{MAG}}$, centred at $900 \mathrm{mV}$ for $90^{\circ}$ phase-shift and $0 \mathrm{~dB}$ power ratio between the signals $u_{1}$ and $u_{2}$. These transfer functions are re-centred around $0 \mathrm{mV}$ with additional differential amplifiers with appropriate voltages at the reference inputs $V_{\text {ref1 }}$ and $V_{\text {ref2 }}$, obtained from a very stable and low noise voltage reference; this allows providing an additional amplification of signals $V_{\mathrm{PHS}}$ and $V_{\mathrm{MAG}}$.

Wide bandwidth operational amplifiers OPA1-4 are used to isolate the sensor and the reference network $R_{C^{-}} C_{C}$ from the rest of the circuit. At motional series resonance frequency (MSRF) the sensor reduces to a motional resistance $R_{m}$ in parallel with the so-called static capacitance $C_{0}$; therefore for optimum operation it is convenient to select $R_{C}$ and $C_{C}$ similar to $R_{m}$ and $C_{0}$, respectively. Effectively, under these conditions and at the MSRF of the sensor, the voltages $u_{\varphi}$ and $u_{A}$ corresponding to the phase-shift and to the decibel ratio of the input signals $u_{1}$ and $u_{2}$, respectively, should be, ideally, zero; this provides a way to calibrate the system.

Additionally, far from resonance the sensor behaves like the parallel capacitance $C_{0}$, and the network formed by the resistance $R_{t}$ and the sensor reduces to a low-pass filter $R_{t}-C_{0}$ of very high cutoff frequency around several megahertz. Consequently slow phase noises in the input testing signal are equally transferred to both branches and eliminated by the differential system, and then improving the stability.

\section{MATERIALS AND METHOD}

\section{A. Sensors and accessories}

$10 \mathrm{MHz}$ fundamental frequency AT-cut quartz sensors, with $13.67 \mathrm{~mm}$ blank diameter and $5.11 \mathrm{~mm}$ of $\mathrm{Cr} / \mathrm{Au}$ electrode diameter ( $100 \AA$ of $\mathrm{Cr}$ and $1000 \AA$ of $\mathrm{Au}$ ), were used in the experiments. Two home-made cells were used, one with a volume capacity of $200 \mu \mathrm{l}$ for the experiments done inbatch with different concentrations of glycerol in water, described elsewhere, ${ }^{42}$ and a different one for the experiments in flow with $30 \mu \mathrm{l}$ volume capacity (Fig. 4). Other instruments associated with the experiment were: Impedance Analyzer HP4291A, frequency meter HP53181A, multimeter 


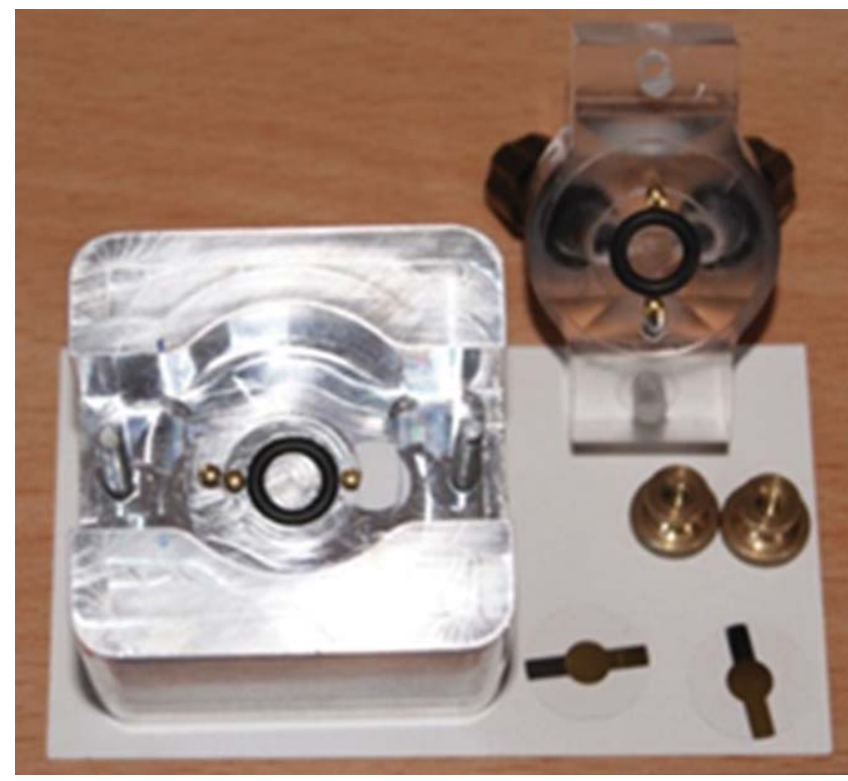

FIG. 4. (Color online) Flow cell.

HP34401A, RF signal generator model HP8664A, and data acquisition system AWSense IP/AQ-1 from AWSensors Inc.

\section{B. Circuit implementation}

The circuits for both systems were made following recommendations applicable to high frequency layout $\operatorname{design}^{63,64}$ and implemented in four-layer surface-mount technology. The following ICs for the main parts of the oscillator were used: OPA860 for the OTAs and AD835 for the implementation of the automatic gain control and differential amplification. The value of $R_{C}$ must be appropriately selected to assure the fulfilment of the loop-gain condition, according to Eq. (12), for given values of $A_{D}$ and $k$, and for the expected range of values of $R_{m}$, which mainly depends on the damping media in contact with the sensor. In this particular case a value of $R_{C}=1 \mathrm{~K} \Omega$ has been selected for an $R_{m}$ range between 10 and $1.2 \mathrm{~K} \Omega$, keeping in mind the reachable values of $k$ $(0 \ll 1,2)$ and $A_{D} \approx 1 . L_{C}-C_{C}$ filters have been selected matched and for a resonance at $10 \mathrm{MHz}$ when using $10 \mathrm{MHz}$ AT-cut quartz resonators. The implemented design is shown in Figs. 5(a) and 5(b).

For the phase-shift characterization interface, phaseshifting networks formed by $R_{i}$ and $C_{i}$ were designed for a cut-off frequency $(-3 \mathrm{~dB})$ at $10 \mathrm{MHz}$ in order to obtain two signals $90^{\circ}$ phase-shifted and of similar amplitude; $500 \mathrm{MHz}$ unity-gain bandwidth operational amplifiers based on the IC OPA656 from Texas Instruments were used for OPA1-4; Instrumentation amplifiers based on the IC AD623 from Ana$\log$ Devices were used for a further amplification of the recentred signals $V_{\mathrm{PHS}}$ and $V_{\mathrm{MAG}}$, obtaining a final phase and magnitude amplifications of $100 \mathrm{mV} /{ }^{\circ}$ and $300 \mathrm{mV} / \mathrm{dB}$, respectively. The values for the components of the reference network $R_{C}$ and $C_{C}$ were selected similar, but using standard values, to the motional resistance and parallel capacitance of the $10 \mathrm{MHz}$ sensor under the liquid load conditions presented in the immunosensor application, where phosphate buffered

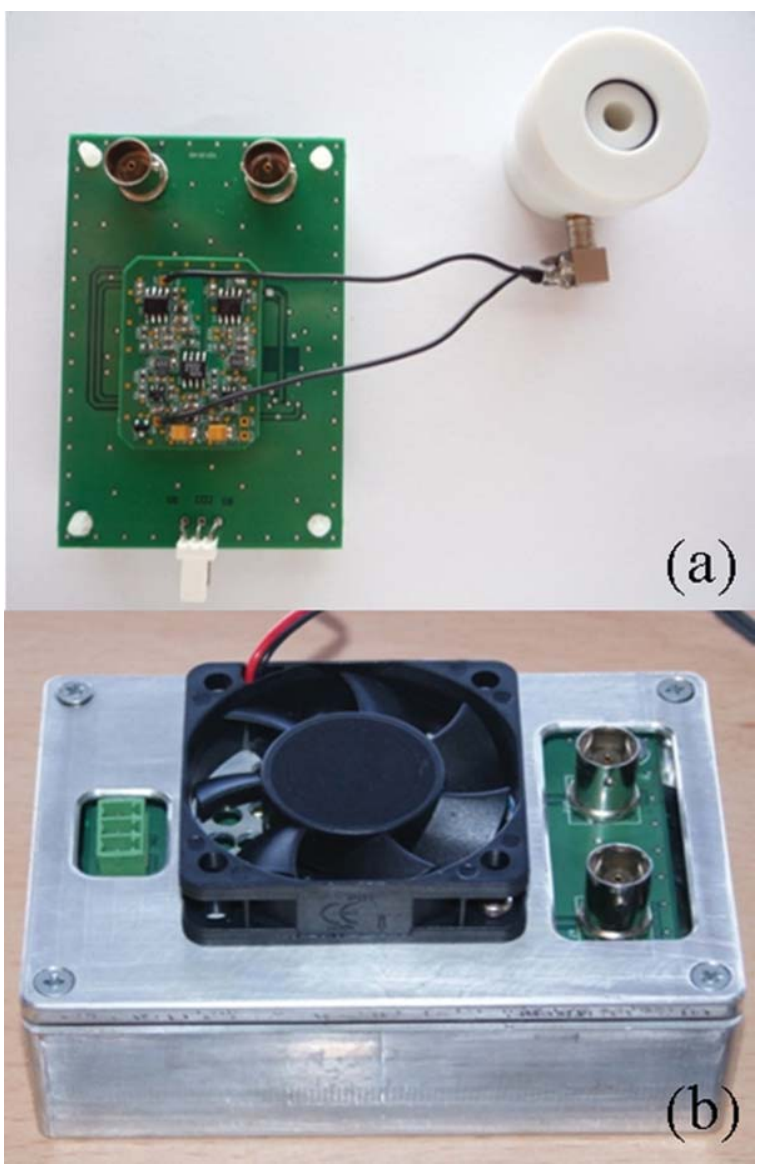

FIG. 5. (Color online) (a) Implemented circuit boards of the oscillator, (b) the final oscillator.

saline (PBS) was used as main working liquid; these values were measured with the IA and standard values were selected for $R_{C}=320 \Omega$ and $C_{C}=10 \mathrm{pF}$; in the experiments $R_{t}$ was selected equal to $R_{C}$. The implemented design is shown in Figs. 6(a) and 6(b).

\section{Experimental methodology}

\section{Calibration and tuning of the phase-shift characterization system}

Before the monitoring, a calibration step of the phaseshift characterization system can be performed easily. In this step an appropriate frequency is selected in the RF generator source $(10 \mathrm{MHz}$, for instance, when using $10 \mathrm{MHz}$ sensors), the sensor is removed and substituted with a $R_{C}-C_{C}$ reference network, in such a way that both differential branches in the system are identical, and the signals at the inputs of the AD8302 should be, ideally, of the same amplitude and phaseshifted $90^{\circ}$. Under this configuration the voltages $V_{\text {ref1 }}$ and $V_{\text {ref2 }}$ are varied for setting the outputs $u_{\varphi}$ and $u_{\mathrm{A}}$ at zero volts; these voltages should be near $900 \mathrm{mV}$. After calibration, the sensor is placed again in its original position and loaded with the working liquid medium, and then the frequency of the RF generator is varied to find again zero volts at the output $u_{\varphi}$. From then on the system is ready for continuous monitoring of the voltages $u_{\varphi}$ and $u_{A}$, which for small sensor resonant 


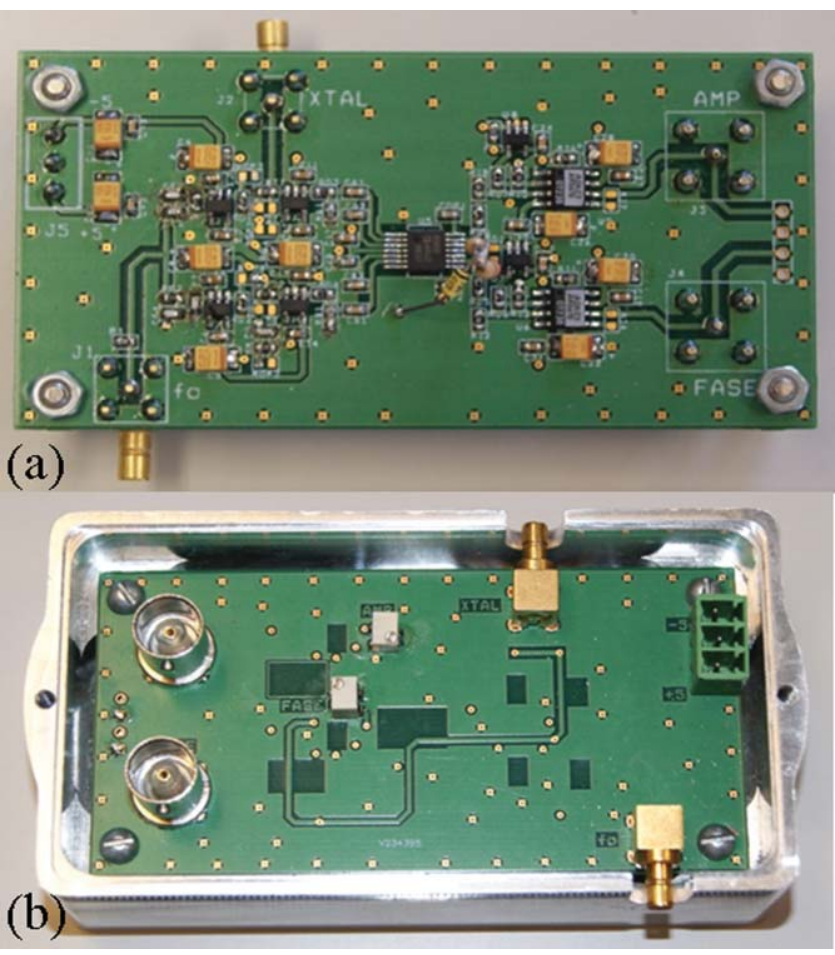

FIG. 6. (Color online) Implemented phase-shift system: (a) upper side, (b) lower side in the customized box.

frequency changes can be directly related to the sensor phaseshift and damping, respectively.

\section{Performance of the oscillator in liquid media}

The compensation of the parallel capacitance of the resonator is useful under heavy load conditions. ${ }^{42}$ In order to test the effective compensation of the parallel capacitance, measurements of the oscillating frequency and the voltage $\mathrm{k}$ associated with the motional resistance $R_{m}$ in the oscillator were made, using liquid solutions of different volume concentrations of glycerol in water $(0 \%, 5 \%, 15 \%, 25 \%$, $35 \%$, and $45 \%$ ); this allows covering a density-viscosity product $(\rho \eta)$ ranged from 1 to $5.2 \mathrm{~kg} \mathrm{~Pa} \mathrm{~s}^{-1}$; data for the density and viscosity of the different solutions were taken from Weast and Astle (1980). ${ }^{65}$ Different compensation capacitors $C_{v}$ were used, with values around the expected parallel capacitance of the sensor $(6.8,10$, and $15 \mathrm{pF})$, to evaluate the parallel capacitance compensation effect. The oscillating frequency and the voltage $\mathrm{k}$ associated with the motional resistance $R_{m}$ were compared with the maximum conductance frequency and the reverse of the conductance peak at resonance obtained from the impedance analyzer with the different liquids. For a complete analysis of the sensor, the conductance, susceptance, impedance phase and impedance modulus plots, and the equivalent parameters $R_{m}, L_{m}, C_{m}$, and $C_{0}$, obtained with the impedance analyzer around the resonance bandwidth, were registered for the sensors in all the liquids and in air, this last taken as reference. Frequency shifts related to the frequency in air were taken with the oscillator and the impedance analyzer for each liquid: the frequency shift in the oscillator $\Delta f_{\mathrm{OSC}}=f_{\mathrm{OSC}}$ (liquid) $-f_{\mathrm{OSC}}$ (air) and the

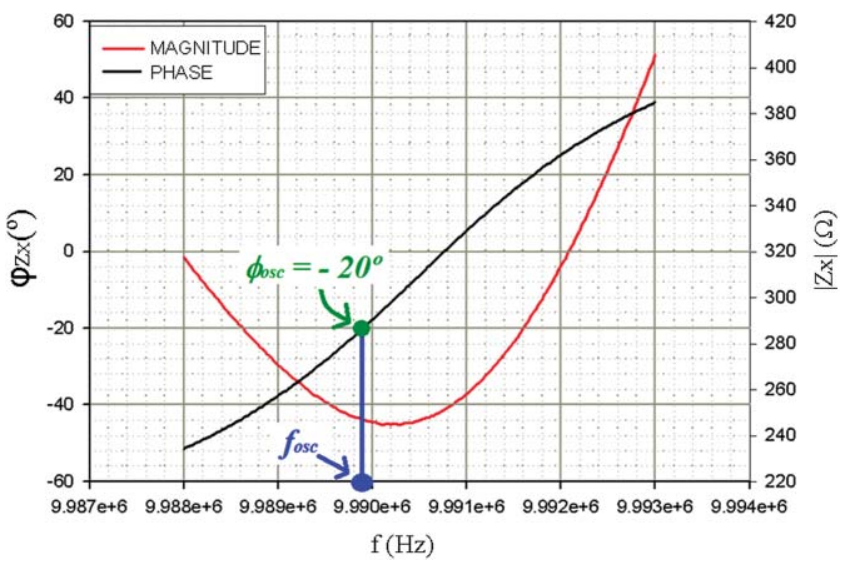

FIG. 7. (Color online) Graphical representation of the impedance phase of the sensor from the plot of the impedance magnitude, knowing the oscillation frequency.

frequency shift obtained from the impedance analyzer $\Delta f_{\text {IA }}$ $=f_{\mathrm{IA}}\left(\mathrm{G}_{\max }-\right.$ liquid $)-f_{\mathrm{IA}}\left(\mathrm{G}_{\max }-\right.$ air $)$, where $\mathrm{G}_{\max }$ indicates the frequency at maximum conductance. The phase of the sensor at the oscillating frequency was obtained starting from the impedance phase plot, obtained with the impedance analyzer for the sensor in contact with each liquid, as depicted in Fig. 7. With the aim of performing a detailed study of the compensation effect of the differential branch formed by the OTA2 and the capacitor $C_{v}$, all the measurements, which were repeated three times and averaged, were made as well under three different configurations of the oscillator: (a) the oscillator without compensation branch (Fig. 8), (b) oscillator with the compensation branch and without the capacitor $C_{v}$ (the same as Fig. 2 without $\mathrm{C}_{V}$ ), and (c) oscillator with different values of $C_{v}$ (Fig. 2). Results are presented and detailed discussed in a subsequent paragraph.

\section{Immunosensor}

A comparison between the classical technique based on frequency shift monitoring and the new one based on phase shift monitoring, using the systems described, under the same

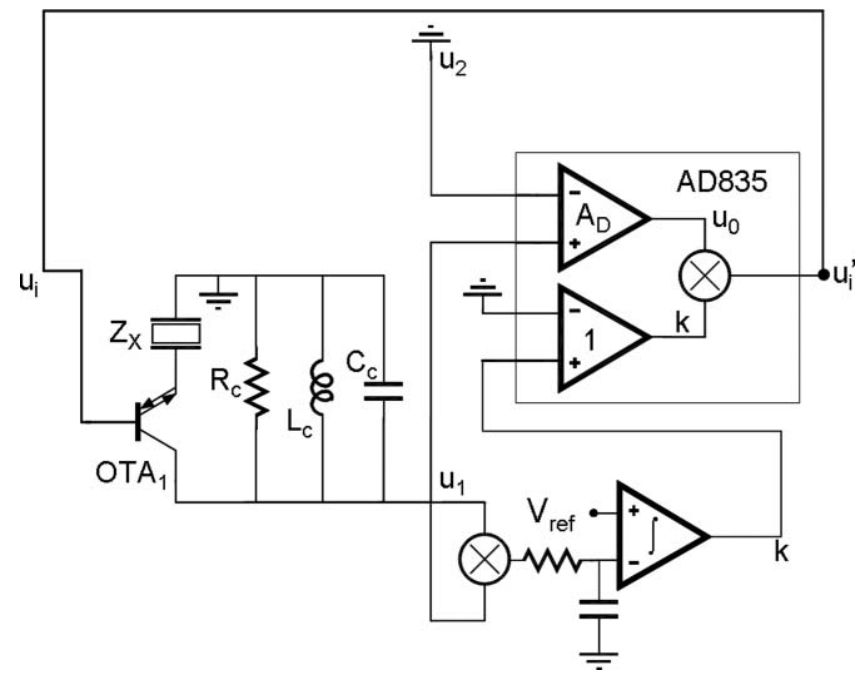

FIG. 8. Oscillator configuration without the differential branch. 
experimental conditions, was performed by implementing a real application based on the detection of low molecular weight pollutants; only with this purpose, a piezoelectric immunosensor for the detection of the pesticide carbaryl, as a validation model, was developed. The following protocol described elsewhere was followed: ${ }^{3}$ the AT-cut quartz crystals were functionalized by immobilizing BSA-CNH carbaryl hapten conjugate on the sensor surface through the formation of a thioctic acid self-assembled monolayer. The crystal was placed in a custom-made flow cell (Fig. 4) and included in a flow-through setup, controlled by a peristaltic pump (Minipuls 3, Gilson), with the injection loop and solutions at the input of the flow cell exchanged by manual Rheodyne valves (models 5020 and 5011, Supelco), according to the flow system described elsewhere. ${ }^{66}$ The whole fluidic system and the sensor characterization circuit with the sensor cell were placed in a custom made thermostatic chamber and all the experiments were performed at $25^{\circ} \mathrm{C} \pm 0.1^{\circ} \mathrm{C}$. To avoid unwanted disturbances the chamber was placed on an antivibration table.

The immunoassay developed to determine carbaryl was an inhibition test based on the conjugate coated format, in which the hapten-conjugate was immobilized on the sensor surface. A fixed amount of a specific monoclonal antibody was mixed with standard solutions of the analyte and pumped over the sensor surface. Since the analyte inhibits antibody binding to the immobilized conjugate, increasing concentrations of analyte will reduce the phase shift induced on the piezoelectric sensor and the corresponding demodulated voltage.

Different standard concentrations of carbaryl were prepared by serial dilutions in PBS, from a $1 \mathrm{mM}$ stock solution in dimetylformamide at $-20{ }^{\circ} \mathrm{C}$. The standards were mixed with a fixed concentration of the monoclonal antibody LIB-CNH45 (from I3BH-UPV (Ref. 67)) in PBS. Analyteantibody solutions were incubated for $1 \mathrm{~h}$ at ambient temperature, then loaded $(250 \mu \mathrm{l})$ into the injection loop in the chamber, and finally, when tempered, injected onto the sensor surface. The phase-shift was monitored in real-time for each analyte concentration during $12 \mathrm{~min}$, as the binding between free antibody and the immobilized conjugate took place. Regeneration of the sensing surface was performed using diluted hydrochloric acid, $0.1 \mathrm{M} \mathrm{HCl}$, to break the antibody-hapten linkage, at a flow rate of $280 \mu \mathrm{l} / \mathrm{min}$ for $4 \mathrm{~min}$, and then with the working buffer solution - phosphate buffered saline $-0.005 \%$ tween 20 (PBST) - for 2 min at the same flow rate. Stabilization of the initial signal was achieved again at a flow rate of $30 \mu \mathrm{l} / \mathrm{min}$ for $2 \mathrm{~min}$. A complete assay cycle took $20 \mathrm{~min}$. This protocol was performed with both characterization systems and the results are discussed in a subsequent paragraph.

\section{RESULTS AND DISCUSSION}

\section{A. Performance of the oscillator in liquid media}

\section{Results}

A linear correlation was found between the motional resistance $R_{m}$ values, obtained from the impedance analyzer, and the voltage $\mathrm{k}$ values, obtained from the oscillator, with a correlation coefficient higher than 0.998 in all the cases. Average deviations between the value of $R_{m}$ obtained from the lineal regression, under the restricted condition of $R_{m}$ being 0 for $k=0$, to be coherent with Eq. (12), and those obtained from the IA, were smaller that $5.5 \%$, reaching the value of $1.1 \%$ for the highest values of $R_{m}$; a uniform reduction of the deviation is observed as the $R_{m}$ values increase. This can be explained taking into account that the OTA1 operates at higher current gains for smaller liquid loads, and that the operation of the amplifier gets farther from the ideal behaviour as the gain increases.

Figure 9(a) collects the frequency shifts obtained with the oscillator $\left(\Delta f_{\mathrm{OSC}}=f_{\mathrm{OSC}}\right.$ (liquid) $-f_{\mathrm{OSC}}($ air $\left.)\right)$ and the impedance analyzer $\left(\Delta f_{\mathrm{IA}}=f_{\mathrm{IA}}\left(\mathrm{G}_{\max }-\right.\right.$ liquid $)-f_{\mathrm{IA}}\left(\mathrm{G}_{\max }\right.$ -air)) for all the liquids and with the different circuit setups and compensation capacitors $C_{v}$. Figure 9(b) shows the impedance working phase of the sensor, obtained as explained, under the different liquid loads and for the different oscillator set-ups and compensation capacitors $C_{v}$ depicted in Fig. 9(a). The $R_{m}$ values in Figs. 9(a) and 9(b) are only included as reference and are near but not necessarily equal to the corresponding $R_{m}$ values obtained from the impedance analyzer with the corresponding liquids; however these values for $R_{m}$ were used in the numerical calculations performed in the discussion below.

The main objective of using the balanced-bridge oscillator is the parallel capacitance compensation under the ideal assumptions made in Sec. IV A. The results shown in Figs. 9(a) and 9(b) allow making the following remarks:

(a) If the parallel capacitance was compensated with an appropriate value of compensation capacitance $C_{v}$, the oscillation frequency shift should be in coincidence, or at least to be very near to the MSRF shift given by the impedance analyzer; however the results depicted in Fig. 9(a) show that none of sets of the oscillator frequency shifts for the different liquids fits the MSRF shifts of the analyzer, only the set of frequency shifts corresponding to the compensation capacitor $C_{v}$ $=6.8 \mathrm{pF}$ seems to be parallel to the results obtained with the impedance analyzer which are taken as reference. The averaged value of the static parallel capacitance $C_{0}$ obtained from the impedance analyzer for all the liquids was $7.37 \mathrm{pF}$, with a maximum deviation of $1.5 \%$, it is to say very near to $6.8 \mathrm{pF}$. This could make to think that a kind of compensation occurs; however this is not reasonable by taking into account the following remark.

(b) According to Fig. 9(b), the sensor working phase for $C_{v}$ $=6.8 \mathrm{pF}$ shows nearly a constant working phase for the whole range of liquid loads (average of $-28^{\circ}$ with a deviation of $0.4^{\circ}$ ). This is in contradiction with the tracking of the MSRF, since different sensor phases correspond to different MSRFs associated with different liquid loads.

In any case, the behaviour depicted in Figs. 9(a) and 9(b) should be explained in the following aspects:

(a) Why is there a sensor working phase at which the oscillation frequency shifts in the working liquid 

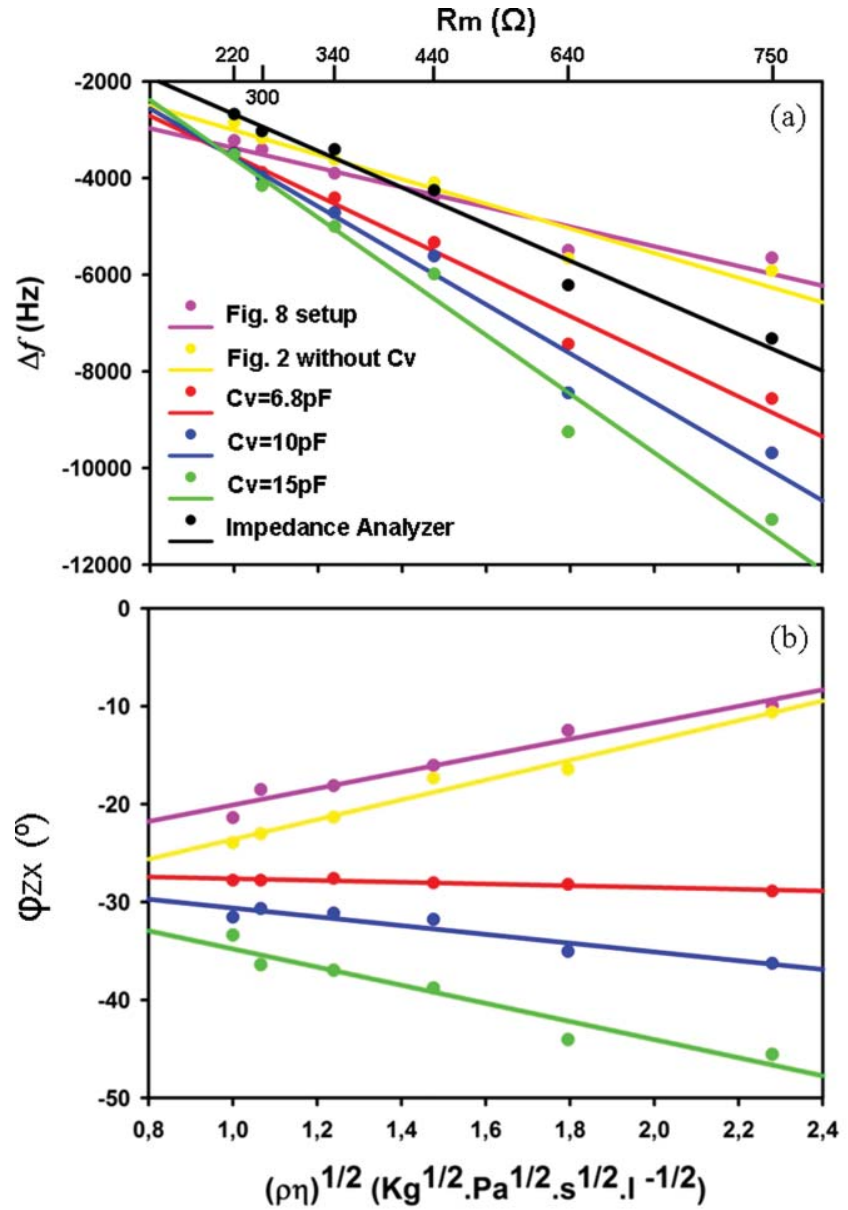

FIG. 9. (Color online) (a) Frequency shifts measured with the oscillator and the impedance analyzer between the sensor in contact with liquid and in contact with air under different liquids, configuration setups, and compensation capacitors. The frequency shifts measured with the IA were taken at conductance peak; (b) phase of the sensor impedance under the different conditions depicted in (a).

solutions, with regard to air, have a parallel behaviour to those associated with the MSRF shifts, obtained from the impedance analyzer?

(b) Why does exist, in the proposed balanced-bridge oscillator, a compensation capacitance $C_{v}$ at which the working phase of the sensor in the oscillator is nearly constant for a relative wide range of liquid loads? Moreover, is there some relation between this value of compensation capacitance and the static parallel capacitance of the sensor $C_{0}$, or the very close values are only a coincidence?

\section{Discussion}

In relation to the first question previously posed, some authors have already described the fact that there are certain working phases of the sensor at which the obtained frequency shifts with regard to the sensor in air have a similar trend to those obtained for the MSRF shifts, depending on the range of loads considered. ${ }^{33,35,68,69}$ Figures 10 (a) and 10(b) show this statement. In Fig. 10(a) the frequency shifts of the sensor under different loading conditions, with regard to the sensor in air, are plotted together with the MSRF shifts

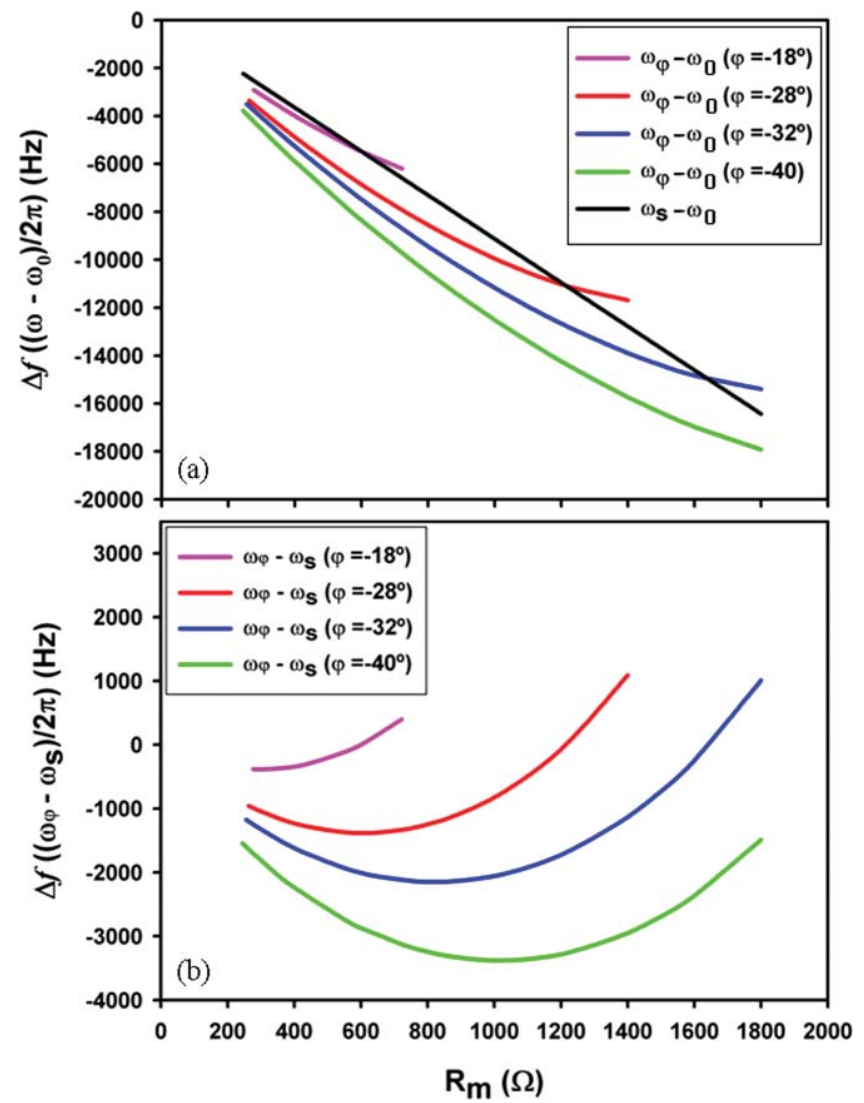

FIG. 10. (Color online) (a) Comparison made by numerical simulation among the frequency shifts corresponding to a certain phase of the sensor impedance, with respect to the frequency of the unperturbed sensor $\left(\Delta \omega_{\varphi}\right.$ $\left.=\omega_{\varphi}-\omega_{0}\right)$, and the motional series resonant frequency shifts under the same conditions $\left(\Delta \omega_{\mathrm{s}}=\omega_{\mathrm{s}}-\omega_{0}\right)$; (b) Frequency deviation between the frequency of the sensor under a certain phase of the sensor impedance and the motional series resonance frequency $\left(\Delta \omega_{\varphi \mathrm{s}}=\omega_{\varphi}-\omega_{\mathrm{s}}\right)$.

for the same loading conditions; in Fig. 10(b) the difference between the frequency shifts, at a given phase of the sensor, and the MSRF shifts in Fig. 10(a) are shown for a better visualization. The plots in Fig. 10(a) have been obtained through the corresponding mathematic equations which are derived in the Appendix for non-disturbing the attention of the reader. The plots have been obtained for different phases of a sensor with a $10 \mathrm{MHz}$ series resonance frequency in air, with different $R_{m}$ associated with the different loads and with the constant motional and static capacitances of $31.5 \mathrm{fF}$ and $7.37 \mathrm{pF}$, respectively, obtained with the sensor used in this work. As can be observed in Fig. 10(a), and previously discussed from other authors, different working phases of the sensor are more appropriate for certain ranges of loads and, additionally, can extend the operation range of the oscillator. Thus, for extending the application to heavy loads a working phase between $-30^{\circ}$ and $-40^{\circ}$ is advisable. It can be observed as well that for a range of loads between 300 and $600 \Omega$ of $R_{m}$, a working phase of $-28^{\circ}$ provides a frequency shift approximately parallel to the MSRF shift, which is the behaviour observed in our experiment. The question now is: why does it occur for a compensation capacitance of $C_{v}$ $=6.8 \mathrm{pF}$. In order to clarify this aspect a deeper analysis of the proposed oscillator shown in Fig. 2 is necessary, which is derived next. 
Voltages $u_{1}$ and $u_{2}$ in the circuit depicted in Fig. 2 can be mathematically expressed in polar form as follows, where the phase of the input voltage $u_{i}$ is taken as zero reference:

$$
\begin{gathered}
u_{1}=u_{i}\left|Y_{X}\right|\left|Z_{R L C}\right|_{\left\langle\varphi_{O T A 1}+\varphi_{R L C}-\varphi_{Z X}\right\rangle}, \\
u_{2}=u_{i}\left|j \omega C_{v}\right|\left|Z_{R L C}\right|_{\left\langle\varphi_{O T A 2}+\varphi_{R L C}+\pi / 2\right\rangle},
\end{gathered}
$$

where $\varphi_{\text {OTA } 1}$ is the phase shift provided by the OTA $1, \varphi_{\text {OTA }}$ is the phase shift given by the OTA2, $\varphi_{R L C}$ is the phase shift provided by the $R_{C^{-}} L_{C}-C_{C}$ parallel branch, $\varphi_{Z X}$ is the phase shift associated with the sensor impedance $Z_{X}, Y_{X}=1 / Z_{X}$ is the admittance of the sensor, $Z_{R L C}$ is the impedance of the $R_{C^{-}} L_{C} C_{C}$ parallel branch, and $\mathrm{j} \omega C_{v}$ is admittance of the compensation capacitor $C_{v}$.

On the other hand, the phase shift associated with the differential amplifier in the AD835 should be very small, because the voltage peak is maintained constant thanks to the $\mathrm{AGC}$, the frequency is always around $10 \mathrm{MHz}$ for all the liquid loads, the differential gain $A_{D}$ is $0 \mathrm{~dB}\left(A_{D}=1\right)$, and the differential error phase is very small ${ }^{70}$ therefore, under the configuration setup depicted in Fig. 8, where the differential branch associated with the OTA2 has been removed, the voltage $u_{i}^{\prime}$ can be expressed as follows:

$$
u_{i}^{\prime}=u_{i} k\left|Y_{X}\right|\left|Z_{R L C}\right|_{\left\langle\varphi_{O T A 1}+\varphi_{R L C}-\varphi_{Z_{X}}\right\rangle},
$$

where the phase shift associated with the AD835 has been neglected.

Figure 9(a) shows that the sensor phase in the configuration set-up where the differential branch is removed (Fig. 8) changes with the liquid load, as follows (phase shift and the reference $R_{m}$ value are written in couples as: $R_{m}$, $\left.\varphi_{Z X}{ }^{\circ}\right): 220 \Omega,-22^{\circ} ; 300 \Omega,-19^{\circ} ; 340 \Omega,-18^{\circ} ; 440 \Omega$, $-16^{\circ} ; 640 \Omega,-13^{\circ}$; and $750 \Omega,-10^{\circ}$. The phase shift increases with increasing the gain (decreasing the load) as it was expected. Because $u_{i}{ }^{\prime}$ is fed-back to the input, $u_{i}{ }^{\prime}=u_{i}$ and Eq. (14) reduces as follows:

$$
k\left|Y_{X}\right|\left|Z_{R L C}\right|_{\left\langle\varphi_{O T A 1}+\varphi_{R L C}-\varphi_{Z_{X}}\right\rangle}=1 .
$$

Consequently, the loop-phase oscillation condition imposes that $\varphi_{C}=\varphi_{Z X}$, where $\varphi_{C}=\varphi_{\text {OTA } 1}+\varphi_{R L C}$; that is, the phase of the sensor compensates the phase shift due to the rest of the elements of the oscillator. Therefore the different working phases of the sensor depicted in Fig. 9(b), for the configuration setup without differential branch, give us the phase shift value due to the oscillator circuitry, mainly due to the OTA1, for the different liquid loads as follows $\left(R_{m}\right.$, $\left.\varphi_{C}\right): 220 \Omega,-22^{\circ} ; 300 \Omega,-19^{\circ} ; 340 \Omega,-18^{\circ} ; 440 \Omega,-16^{\circ}$; $640 \Omega,-13^{\circ}$; and $750 \Omega,-10^{\circ}$.

When the differential branch is added, the phase shift associated with the OTA2 depends on the value of $C_{v}$, since the gain of OTA2 changes approximately from 0.5 to 1 for values of $C_{v}$ from $6.8 \mathrm{pF}$ to $15 \mathrm{pF}$, respectively; however this phase shift is much smaller than in the OTA1 because the smaller change in the gain. Therefore the voltage $u_{2}$ can be expressed as follows:

$$
u_{2}=u_{i}\left|j \omega C_{v}\right|\left|Z_{R L C}\right|_{\left\langle\varphi_{C}^{\prime}+\pi / 2\right\rangle},
$$

where $\varphi^{\prime}{ }_{C}=\varphi_{\text {OTA2 }}+\varphi_{R L C}$
Under this configuration the voltage $u_{0}$ can be written as follows:

$$
\begin{aligned}
u_{0}= & u_{1}-u_{2}=u_{i}\left(\left|Y_{X}\right|\left|Z_{R L C}\right|_{\left\langle\varphi_{O T A 1}+\varphi_{R L C}-\varphi_{Z_{X}}\right\rangle}\right. \\
& \left.-\left|j \omega C_{v}\right|\left|Z_{R L C}\right|_{\left\langle\varphi_{C}^{\prime}+\pi / 2\right\rangle}\right)
\end{aligned}
$$

or alternatively:

$$
u_{0}=\left|u_{0}\right|_{\langle\varphi\rangle},
$$

where $\left|u_{0}\right|=\mathrm{A}^{2}+\mathrm{B}^{2-} 2 \mathrm{AB} \cos (\beta-\alpha)$ and $\tan \varphi=(\mathrm{A} \sin \alpha$ - $\mathrm{B} \sin \beta) /(\mathrm{A} \cos \alpha-\mathrm{B} \cos \beta) ; \mathrm{A}, \mathrm{B}, \alpha$ and $\beta$ being: $A$ $=\left|u_{i}\left\|Y_{X}\right\| Z_{R L C}\right|, B=\left|u_{i}\left\|\mathrm{j} \omega C_{v}\right\| Z_{R L C}\right|, \alpha=\varphi_{C}-\varphi_{Z X}$ and $\beta$ $=\varphi_{C}^{\prime}+\pi / 2$.

Therefore $u_{i}^{\prime}=u_{i}=k\left|u_{0}\right|_{<\varphi>}$ and the loop-phase condition establishes that $\varphi$ must be zero for oscillation. Consequently, now it is possible to check if there is a value of $C_{v}$ which makes $\tan \varphi=0$ for a relatively constant value of the sensor phase for all the liquid loads in the working range. For that the following expression must be fulfilled:

$$
\tan \varphi=\frac{\sin \alpha-\frac{B}{A} \sin \beta}{\cos \alpha-\frac{B}{A} \cos \beta}=0,
$$

where $B / A=\omega C_{v} /\left|Y_{X}\right|$.

The modulus of the admittance $\left|Y_{X}\right|$ and the value of the reactance $X_{m}$ depend on the phase of the sensor and can be written, as a function of the admittance phase of the sensor $\varphi_{Y X}=-\varphi_{Z X}$ and $R_{m}$ as follows (derived from Eqs. (A1) and (A2) in the Appendix):

$$
\begin{gathered}
\left|Y_{X}\right|=\frac{R_{m} \sqrt{1+\tan ^{2} \varphi_{Y_{X}}}}{R_{m}^{2}+X_{m}^{2}}, \\
X_{m}=\frac{\frac{1}{\omega C_{0}}-\sqrt{\left(\frac{1}{\omega C_{0}}\right)^{2}+4\left(\frac{R_{m} \tan \varphi_{Y_{X}}}{\omega C_{0}}-R_{m}^{2}\right)}}{2} .
\end{gathered}
$$

Now, a numerical calculation can be performed in order to obtain the values of $\varphi_{Z X}=-\varphi_{Y X}$ which make $\varphi=0$ in Eq. (19), for the different liquid loads and for the different values of $C_{v}$. For that, the following data were taken into account:

(a) Values for $\varphi_{C}$ were taken from the results obtained with the configuration set-up without the differential branch (Fig. 8), indicated above, for each reference value of $R_{m}$ associated with a certain liquid load.

(b) The value of $\varphi_{C}^{\prime}$ was assumed to be constant for the different values of $C_{V}$ and equal to the value of $\varphi_{C}$ for a $R_{m}$ $=750 \Omega$, this is $\varphi_{C}^{\prime}=-10^{\circ}$; the reason is because the gains for OTA2 changed approximately from 0.5 to 1 for values of $C_{v}$ from $6.8 \mathrm{pF}$ to $15 \mathrm{pF}$, and the gain for OTA1 with a liquid load corresponding to $R_{m}=750 \Omega$ was 1.3 $\left(\approx R_{C} / R_{m}\right)$; therefore similar phase shifts are expected for similar gains.

(c) The value for the angular frequency $\omega$ was taking for a frequency of $10 \mathrm{MHz}$, and the value for the static capacitance was taken from the averaged values obtained with the impedance analyzer, $C_{0}=7.37 \mathrm{pF}$. 


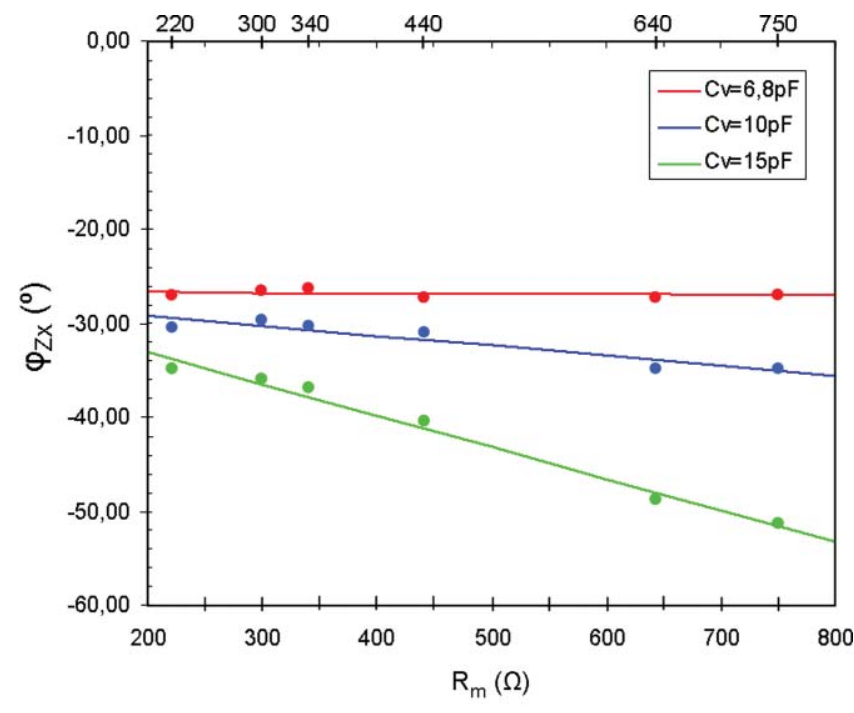

FIG. 11. (Color online) Results of the numerical simulation derived from Eq. (19).

The results of this analysis are presented in Fig. 11. As it can be observed they follow almost the same trend as the ones obtained in Fig. 9(b) for the different values of $C_{v}$; a phase $\varphi_{Z X}=-26.8^{\circ}$ with a deviation of $0.5^{\circ}$, very close to the one obtained in our experiment, results for all the values of $R_{m}$

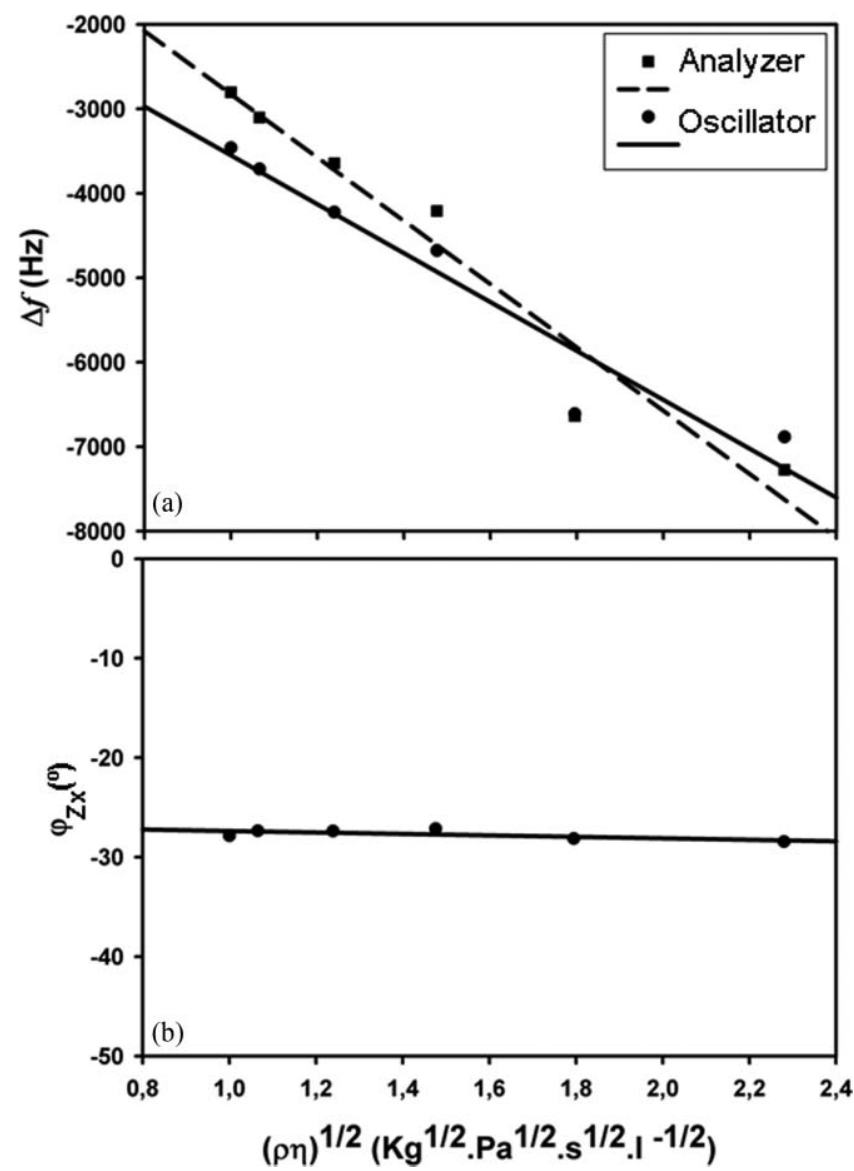

FIG. 12. Results obtained with the oscillator and IA, for a compensation capacitor $C_{v}=6.8 \mathrm{pF}$, under the same conditions as in Fig. 9(a) but with a parallel capacitance of the sensor purposely increased to $12 \mathrm{pF}$ by adding a parallel capacitor.

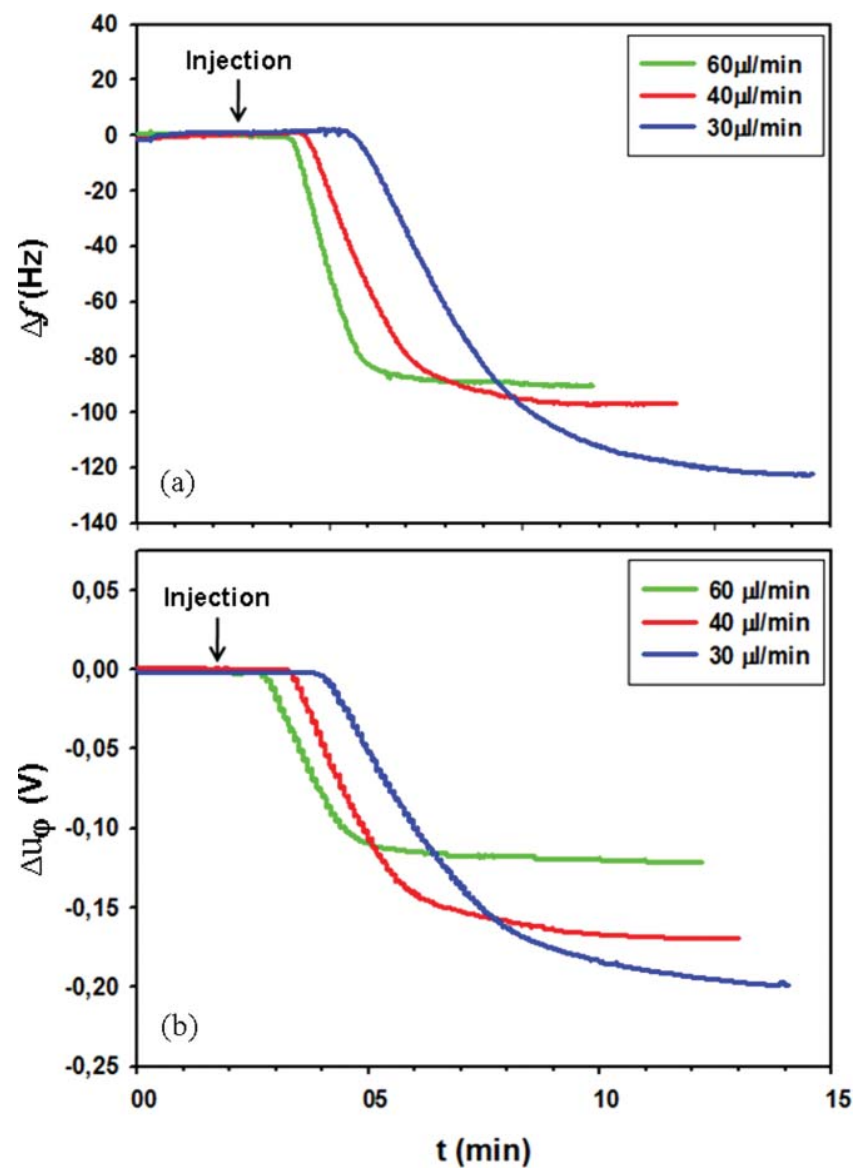

FIG. 13. (Color online) (a) Evolution of the frequency shift in the oscillator, at different flow rates, under the appropriate conditions for maximum signal, and (b) evolution of the voltage associated with the phase-shift in the phaseshift characterization system, at different flow rates, under the appropriate conditions for maximum signal.

with the compensation capacitor $C_{v}=6.8 \mathrm{pF}$, while the sensor phase changes, with the same trend, for a different value of $C_{v}$.

In order to confirm the previous results and to demonstrate that the very close values between the value of $C_{v}$ $=6.8 \mathrm{pF}$, at which frequency shifts for a constant phase of the sensor are nearly parallel to MSRF shifts, and the value of $C_{0}$ was only a coincidence, an additional experiment was performed in which the parallel capacitance of the sensor was changed to $12 \mathrm{pF}$ by an external parallel capacitor. Fig. 12(a) shows how the results for the oscillation frequency shifts leave the parallelism to those from the MSRF shift obtained from the IA, while the sensor phase is maintained relatively constant for all the liquid loads (Fig. 12(b)).

These results indicate that the expected compensation of the parallel capacitance is not provided by the implemented balanced bridge oscillator; the non-ideal behaviour of the oscillator elements and mainly of the OTAs, despite their expected good performance at $10 \mathrm{MHz}$, prevailed on the expected ideal operation of the oscillator circuit. However, the differential branch with the compensation capacitor can be used to compensate the non-ideal behaviour of the oscillator circuit, in such a way that the phase of the sensor can remain relatively constant in a certain range of liquid loads. The value of the capacitor $C_{V}$ for this purpose depends on the sensor and 

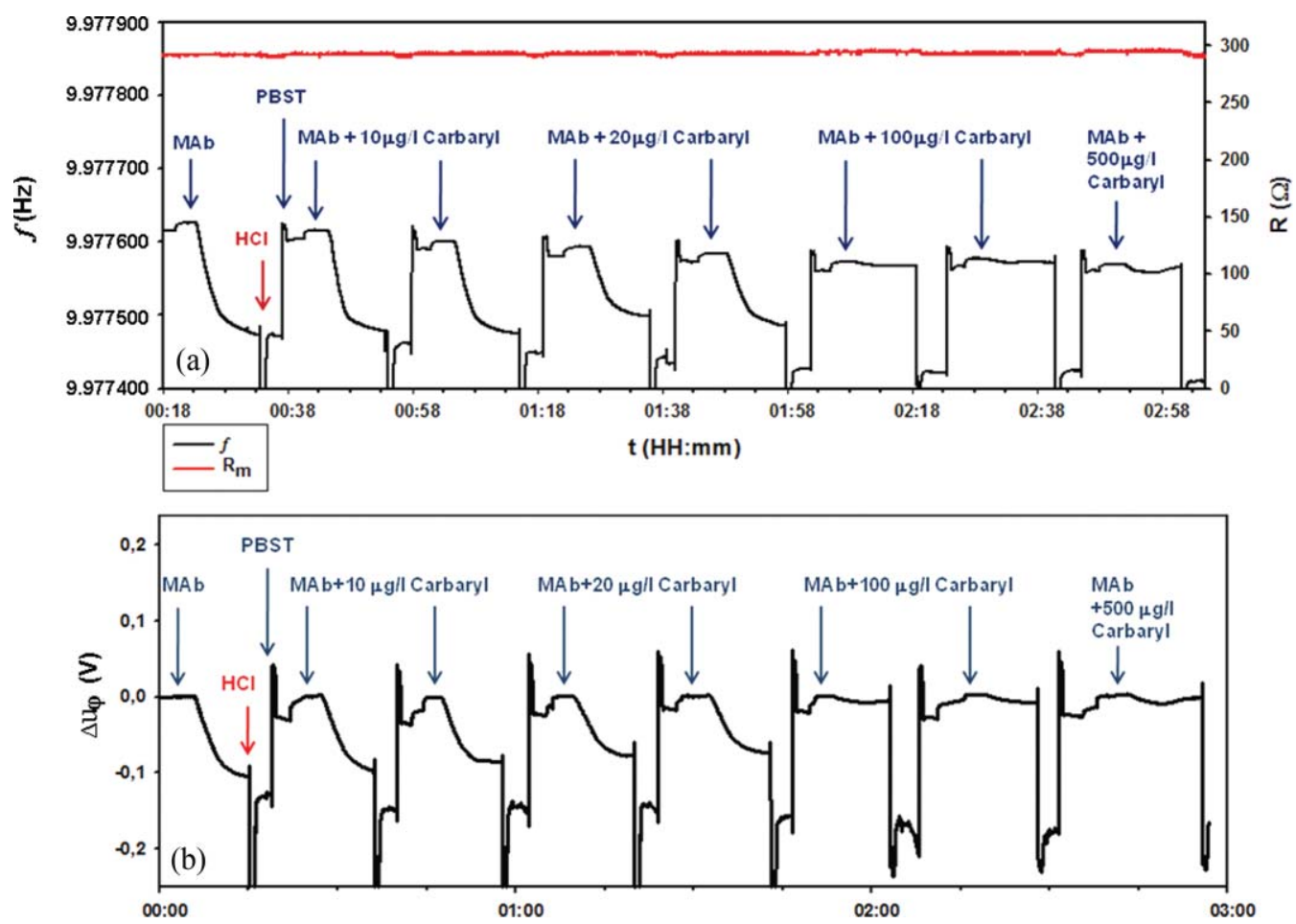

FIG. 14. (Color online) Real time piezoelectric immunosensor response to different concentrations of analyte: (a) with the balanced-bridge oscillator, and (b) with the phase-shift characterization system.

on the behaviour of the oscillator circuit and can be obtained if the phase responses of the OTAs versus the gain are known.

On the other hand, the results show that the oscillator designed can maintain the oscillation under relatively high damping loads, and therefore can be used for frequency shift monitoring in in-liquid microgravimetric applications like, for instance, in piezoelectric biosensors, where the characteristics of the fluid medium remain constant. Therefore the implemented oscillator was used, with $C_{v}=6.8 \mathrm{pF}$, for continuous monitoring the resonance frequency shift of the sensor in the immunosensor application performed to validate the phaseshift characterization method.

\section{B. Immunosensor}

\section{Results}

Figures 13(a) and 13(b) show the real time monitoring of different experiments made with both systems, with the aim of optimizing the flow rate under maximum signal condition; i.e., when the sample is a solution of a reference antibody concentration without antigen. ${ }^{3}$ Because the flow cell was the same in both cases, and the optimized flow rate depends on the cell volume, the same speed of $30 \mu \mathrm{l} / \mathrm{min}$ was obtained for both systems. As it can be observed a very close response was found for both systems keeping in mind the different magnitudes involved. A real time signal of the voltage $u_{\varphi}$, associated with the phase-shift, showed an exponential decay as soon as the molecular interaction occurred after the sample injection; a similar behaviour was observed when the resonant frequency shift was monitored.

Figures 14(a) and 14(b) show a comparison between the real-time signals obtained for the piezoelectric immunosen- sor, for the same experiment, with the frequency-shift (Fig. 14(a)) and phase-shift (Fig. 14(b)) monitoring systems. During the experiments, different concentrations of pesticide in the sample were tested after cyclic regeneration stages described. Only a representative part of the signals obtained in the immunoassay, corresponding to analyte concentrations of 10, 20,100, and $500 \mu \mathrm{g} / \mathrm{l}$, are shown. As it can be observed the resistance $R_{m}$ (voltage k) measured with the oscillator technique (Fig. 14(a)) remained constant as expected in these applications.

\section{Discussion}

These results validate the new characterization concept and the implemented interface. Moreover, a reduction of the noise in the new system was observed as well. Effectively, the noise level in the oscillator technique was of $2 \mathrm{~Hz}$ for a maximum signal of $137 \mathrm{~Hz}$, while for the phase-shift interface was of $1 \mathrm{mV}$ for a maximum signal of $200 \mathrm{mV}$, this indicates an improvement of three times the maximum signal to noise ratio. Furthermore, it is important to notice that this improvement has been got even with relative low frequency sensors (10 MHz), where electronic components and circuits have a very good performance in both, the oscillator and the phasedetector system. Therefore a much more significant improvement is expected to be found with very high fundamental frequency resonators.

\section{CONCLUSIONS AND FUTURE LINES}

A new characterization concept, particularly useful for high resolution QCM applications, based on the phase-shift 
monitoring has been compared with the classical concept of frequency shift monitoring. A balanced bridge oscillator has been proposed for in-liquid QCM applications and proved to be valid for working with sensors under relatively heavy loading conditions. It has been demonstrated that the non-ideal behaviour of the active components which form part of the oscillator prevent making any preliminary ideal theoretical presumption of the expected performance under real conditions; however, despite of the non-ideal behaviour of the oscillators they can follow being used for QCM applications under liquid conditions, and specially for relatively low frequency resonators. Alternatively, the following advantages are expected with the new characterization concept based on the phase-shift monitoring at constant frequency: (a) the sensor is interrogated passively with an external source, which can be designed with high frequency stability an very low phase noise, even at very high frequencies, (b) the sensor circuit is very simple with high level of integration capabilities, (c) the open loop configuration, in contrast to the typical feedback configuration of the oscillator, allows a straightforward noise analysis and minimization, simplifying the design and implementation of the electronics, (d) sensors working at the same fundamental resonance frequency could be characterized, in principle, with only one source, opening the possibility of working with sensor arrays for multianalysis detection.

Following the results presented here, the next step is to perform experiments with the new systems using high fundamental frequency BAW resonators based on inverted mesa technology.

\section{ACKNOWLEDGMENTS}

The authors are grateful to the Spanish Ministry of Science and Technology for the financial support to this research under contract reference AGL2009-13511, and to the company Advanced Wave Sensors S.L. (www.awsensors.com) for the help provided in the development of some parts of this work.

\section{APPENDIX: DERIVATION OF THE FREQUENCY SHIFT BETWEEN THE FREQUENCY OF THE SENSOR AT A CERTAIN PHASE AND THE MSRF}

The admittance of the sensor can be expresses as follows:

$$
\begin{aligned}
Y_{X}= & j \omega C_{0}+\frac{1}{R_{m}+j X_{m}}=\frac{R_{m}}{R_{m}^{2}+X_{m}^{2}} \\
& +j \frac{\omega C_{0}\left(R_{m}^{2}+X_{m}^{2}\right)-X_{m}}{R_{m}^{2}+X_{m}^{2}} .
\end{aligned}
$$

Therefore, the phase of the admittance $\varphi_{Y x}$ complies with the following expression:

$$
\tan \varphi_{Y_{X}}=\frac{\omega C_{0}\left(R_{m}^{2}+X_{m}^{2}\right)-X_{m}}{R_{m}} .
$$

The reactance $X_{m}$ at a certain angular frequency $\omega_{\varphi}$ corresponding to a sensor operating phase $\varphi$ can be written as follows:

$$
\begin{aligned}
X_{m} & =L_{m} \omega_{\varphi}-\frac{1}{C_{m} \omega_{\varphi}}=\left(L_{q}+\Delta L\right) \omega_{\varphi}-\frac{1}{C_{q} \omega_{\varphi}} \\
& =\Delta L \omega_{\varphi}+\frac{1}{C_{q} \omega_{\varphi}}\left(\frac{\omega_{\varphi}^{2}}{\omega_{0}^{2}}-1\right),
\end{aligned}
$$

where it has been assumed that the change in the reactance due to the liquid load is due to an inertial effect associated with the increase in the motional inductance $\Delta L \omega$, the motional capacitance is assumed to be constant and equal to the value in the unperturbed state, $C_{q} ; L_{q}$ is the motional inductance in air; and $\omega_{0}=1 /\left(L_{q} C_{q}\right)^{1 / 2}$ is the resonance angular frequency in the unperturbed state (air).

For liquid loads $\Delta L \omega \approx R_{m}$, assuming the motional resistance in air is small, and the former equation can be approximated as follows:

$$
X_{m} \approx R_{m}+\frac{2}{C_{q} \omega_{0}^{2}} \Delta \omega_{\varphi},
$$

where $\Delta \omega_{\varphi}=\omega_{\varphi}-\omega_{0}$.

By using Eq. (A4) in Eq. (A2), the following relationship is obtained, which relates $\Delta \omega_{\varphi}$, with the sensor admittance phase, the motional resistance $R_{m}$ corresponding to a liquid load, the static parallel capacitance $C_{0}$, the motional capacitance $C_{q}$, and the unperturbed resonance frequency $\omega_{0}$ :

$$
\begin{aligned}
\tan \varphi_{Y_{X}}= & \frac{4 C_{0}}{C_{q}^{2} \omega_{0}^{3} R_{m}} \Delta \omega_{\varphi}^{2}+\frac{4 C_{0} \omega_{0} R_{m}-2}{C_{q} R_{m} \omega_{0}^{2}} \Delta \omega_{\varphi} \\
& +2 \omega_{0} C_{0} R_{m}-1 .
\end{aligned}
$$

The frequency at conductance peak, obtained from IA measurements, corresponds, with negligible error in most of cases, to the MSRF where $X_{m}=0$; therefore, from Eq. (A4), the angular frequency shift corresponding to the MSRF for a certain liquid load with respect to the unperturbed state $\Delta \omega_{s}$ $=\omega_{s}-\omega_{0}$, where $\omega_{s}$ is the angular MSRF, results as follows:

$$
\Delta \omega_{s}=-\frac{C_{q} \omega_{0}^{2} R_{m}}{2}
$$

Consequently, the angular frequency shift between the MSRF and the angular frequency corresponding to a certain sensor admittance phase, $\Delta \omega_{\varphi s}=\omega_{\varphi}-\omega_{s}$, can be obtained from the previous equations as $\Delta \omega_{\varphi s}=\Delta \omega_{\varphi}-\Delta \omega_{s}\left(\Delta f_{\varphi s}\right.$ $\left.=\Delta \omega_{\varphi s} / 2 \pi\right)$. The previous equations have been used, for $10 \mathrm{MHz}$ resonance frequency, $C_{q}=31.5 \mathrm{fF}$ and $C_{0}=7.37$ $\mathrm{pF}$ corresponding to the sensor used, and for different values of the sensor admittance phase, in the derivation of the results shown in Figs. 10(a) and 10(b).

${ }^{1}$ G. Sauerbrey, Z. Phys. 155, 206 (1959).

${ }^{2}$ A. Janshoff, H. J. Galla, and C. Steinem, Angew. Chem., Int. Ed. 39, 4004 (2000).

${ }^{3}$ C. March, J. J. Manclús, Y. Jiménez, A. Arnau, and A. Montoya, Talanta 78, 827 (2009).

${ }^{4}$ M. I. Rocha, C. March, A. Montoya, and A. Arnau, Sensors 9, 5740 (2009).

${ }^{5}$ L. Richert, P. Lavalle, D. Vaultier, B. Senger, F. Stoltz, P. Schaaf, J. C. Voegel, and C. Picart, Biomacromolecules 3, 1170 (2002).

${ }^{6}$ F. Hook, A. Ray, B. Norden, and B. Kasemo, Langmuir 17, 8305 (2001).

${ }^{7}$ I. BenDov, I. Willner, and E. Zisman, Anal. Chem. 69, 3506 (1997). 
${ }^{8}$ M. Nirschl, A. Blüher, C. Erler, B. Katzschner, I. Vikholm-Lundin, S. Auer, J. Vörös, W. Pompe, M. Schreiter, and M. Mertig, Sens. Actuators A 156, 180 (2009).

${ }^{9}$ Y. S. Fung and Y. Y. Wong, Anal. Chem. 73, 5302 (2001).

${ }^{10}$ X. D. Zhou, L. J. Liu, M. Hu, L. Wang, and J. Hu, J. Pharm. Biomed. Anal. 27, 341 (2002)

${ }^{11}$ R. Gabl, M. Schreiter, E. Green, H. D. Feucht, H. Zeininger, J. Runck, W. Reichl, R. Primig, D. Pitzer, G. Eckstein, and W. Wersing, Proc. IEEE Sens. 2, 1184 (2003).

${ }^{12}$ R. Gabl, H. D. Feucht, H. Zeininger, G. Eckstein, M. Schreiter, R. Primig, D. Pitzer, and W. Wersing, Biosens. Bioelectron. 19, 615 (2004).

${ }^{13}$ G. Wingqvist, V. Yantchev, and I. Katardjiev, Sens. Actuators A 148, 88 (2008).

${ }^{14}$ J. Weber, W. M. Albers, J. Tuppurainen, M. Link, R. Gabl, W. Wersing, and M. Schreiter, Sens. Actuators A 128, 84 (2006).

${ }^{15}$ Z. Lin, C. M. Yip, I. S. Joseph, and M. D. Ward, Anal. Chem. 65, 1546 (1993).

${ }^{16}$ J. Bjurstrom, G. Wingqvist, and I. Katardjiev, IEEE Trans. Ultrason. Ferroelectr. Freq. Control 53, 2095 (2006).

${ }^{17}$ G. Wingqvist, J. Bjurstrom, L. Liljeholm, V. Yantchev, and I. Katardjiev, Sens. Actuators B 123, 466 (2007).

${ }^{18} \mathrm{G}$. Wingqvist, H. Anderson, C. Lennartsson, T. Weissbach, V. Yanchtev, and A. Lloyd Spetz, Biosens. Bioelectron. 24, 3387 (2009).

${ }^{19}$ L. A. Francis, J. M. Friedt, R. De Palma, C. Zhou, C. Bartic, A. Campitelli, and P. Bertrand, Frequency Control Symposium and Exposition, 2004. Proceedings of the 2004 IEEE International, pp. 241-249 (2004).

${ }^{20}$ G. L. Harding, Sens. Actuators A 88, 20 (2001).

${ }^{21}$ Z. Wang, J. D. N. Cheeke, and C. K. Jen, Appl. Phys. Lett. 64, 2940 (1994).

${ }^{22}$ K. Kalantar-Zadeh, W. Wlodarski, Y. Y. Chen, B. N. Fry, and K. Galatsis, Sens. Actuators B 91, 143 (2003).

${ }^{23}$ N. Moll, E. Pascal, D. H. Dinh, J. L. Lachaud, L. Vellutini, J. P. Pillot, D. Rebière, D. Moynet, J. Pistré, D. Mossalayi, Y. Mas, B. Bennetau, and C. Déjous, ITBM-RBM 29, 155 (2008).

${ }^{24}$ H. Ogi, H. Nagai, Y. Fukunishi, M. Hirao, and M. Nishiyama, Anal. Chem. 81, 8068 (2009).

${ }^{25}$ A. Arnau, V. Ferrari, D. Soares, and H. Perrot, in Piezoelectric Transducers and Applications, edited by A. Arnau, 2nd ed. (Springer Verlag, Berlin Heidelberg, 2008), ch. 5, pp. 117-186.

${ }^{26}$ F. Eichelbaum, R. Borngräber, J. Schröder, R. Lucklum, and P. Hauptmann, Rev. Sci. Instrum. 70, 2537 (1999).

${ }^{27}$ J. Schröder, R. Borngräber, R. Lucklum, and P. Hauptmann, Rev. Sci. Instrum. 72(6), 2750 (2001).

${ }^{28}$ S. Doerner, T. Schneider, J. Schröder, and P. Hauptmann "Universal impedance spectrum analyzer for sensor applications" in Proceedings of IEEE Sensors 1, pp. 596-594 (2003).

${ }^{29}$ M. Rodahl and B. Kasemo, Rev. Sci. Instrum. 67, 3238 (1996).

${ }^{30}$ M. Rodahl and B. Kasemo, Sens. Actuators B 37, 111 (1996).

${ }^{31}$ C. Barnes, Sens. Actuators A 30(3), 197 (1992).

${ }^{32} \mathrm{~K}$. O. Wessendorf, "The lever oscillator for use in high resistance resonator applications," in Proceedings of the 1993 IEEE International Frequency Control Symposium, pp. 711-717 (1993).

${ }^{33}$ R. Borngräber, J. Schröder, R. Lucklum, and P. Hauptmann, IEEE Trans. Ultrason. Ferroelectr. Freq. Control 49(9), 1254 (2002).

${ }^{34}$ H. Ehahoun, C. Gabrielli, M. Keddam, H. Perrot, and P. Rousseau, Anal. Chem. 74, 1119 (2002).

${ }^{35}$ S. J. Martin, J. J. Spates, K. O. Wessendorf, T. W. Schneider, and R. J. Huber, Anal. Chem. 69, 2050 (1997).

${ }^{36}$ V. Ferrari, D. Marioli, and A. Taroni, IEEE Trans. Instrum. Meas. 50, 1119 (2001).

${ }^{37}$ A. Arnau, T. Sogorb, Y. Jiménez, Rev. Sci. Instrum. 73(7), 2724 (2002).

${ }^{38}$ B. Jakoby, G. Art, and J. Bastemeijer, IEEE Sens. J. 5(5), 1106 (2005).

${ }^{39}$ M. Ferrari, V. Ferrari, D. Marioli, A. Taroni, M. Suman, and E. Dalcanale, IEEE Trans. Instrum. Meas. 55(3), 828 (2006).
${ }^{40}$ M. Ferrari, V. Ferrari, and K. K. Kanazawa, Sens. Actuators A 145, 131 (2008).

${ }^{41}$ C. Riesch and B. Jakoby, IEEE Sens. J. 7(3) 464 (2007).

${ }^{42}$ A. Arnau, J. V. García, Y. Jimenez, V. Ferrari, and M. Ferrari, Rev. Sci. Instrum. 79, 075110 (2008).

${ }^{43}$ C. Barnes, Sens. Actuators A 29(1), 59 (1991).

${ }^{44}$ J. Auge, P. Hauptmann, F. Eichelbaum, and S. Rösler, Sens. Actuators B 18-19, 518 (1994).

${ }^{45}$ J. Auge, P. Hauptmann, J. Hartmann, S. Rösler, and R. Lucklum, Sens. Actuators B 24-25, 43 (1995).

${ }^{46}$ C. Chagnard, P. Gilbert, A. N. Watkins, T. Beeler, and D. W. Paul, Sens. Actuators B 32, 129 (1996).

${ }^{47}$ D. W. Paul and T. L. Beeler, Piezoelectric sensor Q-loss compensation. U.S. Patent No. 4,788,466 (1998).

${ }^{48}$ L. Rodríguez-Pardo, J. Fariña, C. Gabrielli, H. Perrot, and R. Brendel, Sens. Actuators B 103, 318 (2004).

${ }^{49}$ L. Rodríguez-Pardo, J. Fariña, C. Gabrielli, H. Perrot, and R. Brendel, Electron. Lett. 42(18), 1065 (2006).

${ }^{50} \mathrm{~K}$. O. Wessendorf, The active-bridge oscillator for use with liquid loaded QCM sensors. Proceedings of IEEE International Frequency Control Symposium and PDA Exhibition, pp. 400-407 (2001).

${ }^{51}$ E. Benes, M. Schmid, M. Gröschl, P. Berlinger, H. Nowotny, and K. C. Harms, Proceedings of the Joint Meeting of the European Frequency and Time Forum and the IEEE International Frequency Control Symposium, Vol. 2, p. 1023-1026 (1999).

${ }^{52}$ J. Rabe, S. Büttgenbach, B. Zimmermann, and P. Hauptmann, 2000 IEEE/EIA International Frequency Control Symposium and Exhibition, pp. 106-112 (2000).

${ }^{53}$ E. Uttenthaler, M. Schräml, J. Mandel, and S. Drost, Biosens. Bioelectron. 16, 735 (2001).

${ }^{54}$ B. Zimmermann, R. Lucklum, and P. Hauptmann, Sens. Actuators B 76, 47 (2001).

${ }^{55}$ B. P. Sagmeister, I. M. Graz, R. Schwödiauer, H. Gruber, and S. Bauer, Biosens. Bioelectron. 24, 2643 (2009).

${ }^{56}$ E. A. Bustabad, G. García, L. Rodriguez-Pardo, J. Fariña, H. Perrot, C. Gabrielli, B. Bucur, M. Lazerges, D. Rose, C. Compere, and A. Arnau, Sensors, 2009 IEEE, pp. 687-690 (2009).

${ }^{57}$ A. Arnau, Y. Montagut, J. V. García, and Y. Jimenez, Meas. Sci. Technol. 20, 124004 (2009).

${ }^{58}$ S. J. Martin, V. E. Granstaff, and G. C. Frye, Anal. Chem. 63, 2272 (1991).

${ }^{59}$ K. K. Kanazawa and J. G. Gordon II, Anal. Chim. Acta 175, 99 (1985).

${ }^{60}$ D. M. Dress, H. R. Shanks, R. A. Van Deusen, and A. R. Landin, Method and system for detecting material using piezoelectric resonators. U.S. Patent 5,932,953 (1999).

${ }^{61}$ M. Pax, J. Rieger, R. H. Eibl, C. Thielemann, and D. Johannsmann, Analyst 130, 1474 (2005)

${ }^{62}$ Analog Devices, LF-2.7GHz, RF/IF Gain and Phase Detector, AD8302 Data Sheet (2002)

${ }^{63}$ H. Johnson, High-Speed Digital Design: A Handbook of Black Magic (Prentice Hall, Englewood Cliffs, NJ, 1993).

${ }^{64}$ M. Montrose, Emc \& the Printed Circuit Board: Design, Theory, \& Layout Made Simple (IEEE Press, New York, 1998).

${ }^{65}$ R. C. Weast and M. J. Astle, CRC Handbook of Chemistry and Physics, 60th ed. (CRC Press, Inc., Boca Raton, FL, 1980).

${ }^{66}$ A. Montoya, A. Ocampo, and C. March, in Piezoelectric Transducers and Applications, edited by A. Arnau, 2nd ed. (Springer-Verlag, Berlin, Heidelberg, 2008), Ch 12, pp. 289-306.

${ }^{67}$ A. Abad, J. Primo, and A. Montoya, J. Agric. Food Chem. 45, 1486 (1997).

${ }^{68}$ D. Soares, Meas. Sci. Technol. 4, 549 (1993).

${ }^{69}$ C. Fruböse, K. Doblhofer and D. M. Soares, Ber. Bunsenges. Phys. Chem. 97(3), 475 (1993).

${ }^{70}$ Analog Devices, $250 \mathrm{MHz}$, Voltage Output, 4-quadrant multiplier, AD835 Data Sheet (1994-2010). 